\title{
Quantum Channels with Memory
}

\author{
Dennis Kretschmann* and Reinhard F. Werner| \\ Institut für Mathematische Physik, Technische Universität Braunschweig, \\ Mendelssohnstr. 3, D-38106 Braunschweig, Germany
}

(Dated: 9 May 2005)

\begin{abstract}
We present a general model for quantum channels with memory, and show that it is sufficiently general to encompass all causal automata: any quantum process in which outputs up to some time $t$ do not depend on inputs at times $t^{\prime}>t$ can be decomposed into a concatenated memory channel. We then examine and present different physical setups in which channels with memory may be operated for the transfer of (private) classical and quantum information. These include setups in which either the receiver or a malicious third party have control of the initializing memory. We introduce classical and quantum channel capacities for these settings, and give several examples to show that they may or may not coincide. Entropic upper bounds on the various channel capacities are given. For forgetful quantum channels, in which the effect of the initializing memory dies out as time increases, coding theorems are presented to show that these bounds may be saturated. Forgetful quantum channels are shown to be open and dense in the set of quantum memory channels.
\end{abstract}

PACS numbers: 03.67.Hk,03.67.Pp,89.70.+c

Keywords: Quantum memory channels vs. memoryless channels, correlated noise, causal automata, forgetful channels, coding theorems, mutual information, coherent information.

Contents

\author{
I. Introduction \\ A. Outline and Overview \\ B. Model Svstems and Related Work
}

II. Language and Notations

A. States. Channels, and Observables

B. Heisenberg vs. Schrödingen

C. Distance between Quantum Channels

III. Channels with Memory

A. The Constructive Approach

B. Channel Capacity

C. Examples

D. Pure Channels

IV. The Structure of Causal Channels

\section{Forgetful Channels}

VI. Entropic Bounds and Channel Coding

A. Entropic Bounds

B. Coding Theorems for Forgetful Channels

VII. Summary and Outlook

Acknowledgments

Appendix

1. $\mathrm{C}^{*}$-Algebras

2. Quasi-Local Algebras

3. Stinespring's Representation

*Electronic address: d.kretschmann@tu-bs.de

${ }^{\dagger}$ Electronic address: r.werner@tu-bs.de
4. GNS-Representation of Quantum States

\section{INTRODUCTION}

Any processing of quantum information, be it storage or transfer, can be represented as a quantum channel: a completely positive and trace-preserving map $S$ that transforms states (density matrices) on the sender's end of the channel into states on the receiver's end. Until now most of the work on quantum channels has concentrated on memoryless channels, which are characterized by the requirement that successive channel inputs

9 are acted on independently. Mathematically, this means that messages of $n$ symbols are processed by the tensor product channel $S^{\otimes n}$.

However, in many real-world applications the assumption of having uncorrelated noise channels cannot be justified, and memory effects need to be taken into account. It thus seems desirable to extend the theory of quantum channels to encompass memory effects, and to create a common framework in which experiments with both correlated and uncorrelated noise can be naturally described. In fact, such a framework is already necessary for estimates on almost memoryless channels, for instance when assessing whether a particular system can arguably be modelled as a memoryless channel. In the present paper such a unified framework will be presented, and it will be shown how this model can be applied to the description of different information processing tasks, such as (private) classical and quantum information transfer. 

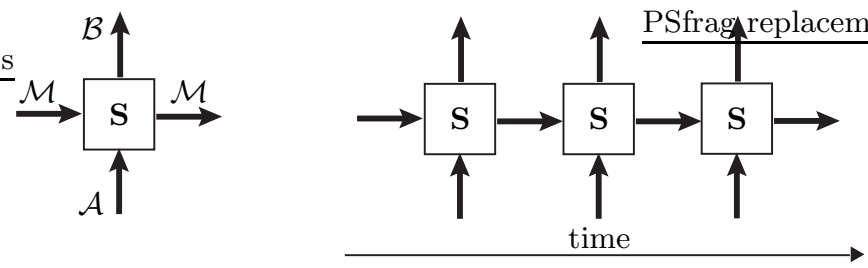

FIG. 1: Left: A quantum memory channel with input register $\mathcal{A}$, output register $\mathcal{B}$, and memory system $\mathcal{M}$. Right: $\mathrm{A}$ threefold concatenation $S_{3}$ of memory channels, with time running from left to right, and coded information running from bottom to top.

\section{A. Outline and Overview}

In our contribution we present a general model for quantum channels with memory. In addition to Alice's input register $\mathcal{A}$ and Bob's output register $\mathcal{B}$, such a channel has an additional memory input and an additional memory output, denoted by $\mathcal{M}$ (cf. Fig. 1] left). Long messages with $n$ signal states will then be processed by subsequent application of these memory channels, resulting in the concatenated channel $S_{n}$ depicted in Fig. 1 (right). This picture will be turned into a rigorous definition in Section IIIA after the mathematical framework will have been introduced in Section III

In such a setup, the memory system is passed on from one application of the channel to the next, and introduces (quantum or classical) correlations between consecutive signal states. If no memory system is present, the concatenated channel will simply be a product channel, bringing us back to the memoryless realm in which consecutive signal states are acted on independently.

This model marks a constructive approach to quantum channels with memory. It is certainly the appropriate framework when the physical realization of the memory $\mathcal{M}$ is known. However, in many applications of information theory only the input-output behavior of a channel is of interest. From this point of view the memory would be part of the internal workings of the channel, and would not be made part of the description. We call this way of describing channels the axiomatic approach: It takes a channel as a transformation turning infinite strings of input systems to infinite strings of outputs, with only two basic assumptions: translation invariance and the condition of causality. Outputs up to some time $t$ do not depend on inputs at times $t^{\prime}>t$. In the classical theory, such channels are sometimes called non-anticipatory. It is clear from Fig. 1 that a channel with memory automatically satisfies this causality condition.

Taking a causal channel and representing it as a channel with memory amounts to reconstructing a model of the channel and its internal memory states and dynamics. This is a highly non-trivial task, even in the classical

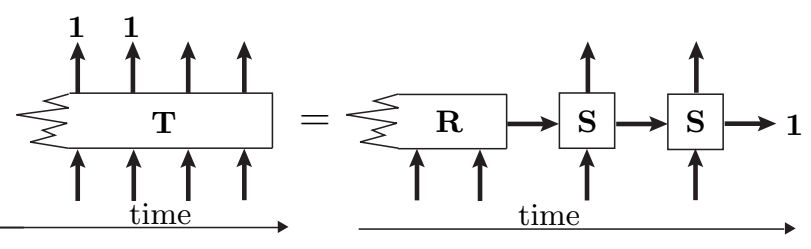

FIG. 2: By the Structure Theorem, a causal automaton $T$ can be decomposed into a chain of concatenated memory channels $S$ plus some input initializer $R$. Evaluation with the identity operator 1 means that the corresponding output is ignored.

case. However, a formal reconstruction can always be given. This is what we call the Structure Theorem for causal channels, and is illustrated in Fig. 2. A rigorous version will be given as Th. 4 in Section IV] In general, it produces not only the channel step operator $S$, but also a map $R$ defining the influence of input states in the remote past on the memory. Intuitively, however, such a map is often not needed, because memory effects decrease in time. A similar condition is needed for passing from the constructive approach of channels with memory to causal input-output channels: Since the constructive approach allows one to choose the initial memory state, output states in general depend on this choice, and in general this influence will depend on the time after initialization. So in order to get a time translation invariant channel without such dependence, the channel $S$ must lose the initialization information. We call $S$ forgetful if outputs at a large time $t$ depend only weakly on the memory initialization at time zero, in a sense made precise in Section D For forgetful channels, memory effects will be shown to decrease even exponentially.

Not every channel is forgetful. The prime counterexample is a channel with a global classical switch discussed in Section IIC The memory in this case is a classical bit, left unchanged by $S$, but determining which of two memoryless channels $S_{0}, S_{1}$ is applied to the input at each time. However, we will show in Section $\nabla$ that generic memory channels are in fact forgetful, in the sense that every non-forgetful quantum channel can be approximated by a forgetful channel to arbitrary degree of accuracy. In addition, for every forgetful quantum channel we may find a finite-size neighborhood in which all channels are likewise forgetful. In mathematical terms, forgetful quantum channels are both open and dense in the set of quantum memory channels.

For quantum channels with memory, capacity can be defined along the lines familiar from the memoryless setting 1, 2], both for the transmission of classical and quantum information. Channel capacity expresses quantitatively how well a given channel $S$ can simulate a noiseless qubit (or bit) channel: roughly speaking, it is the maximal number of ideal qubit (resp. bit) transmissions per use of the channel, taken in the limit of long messages 


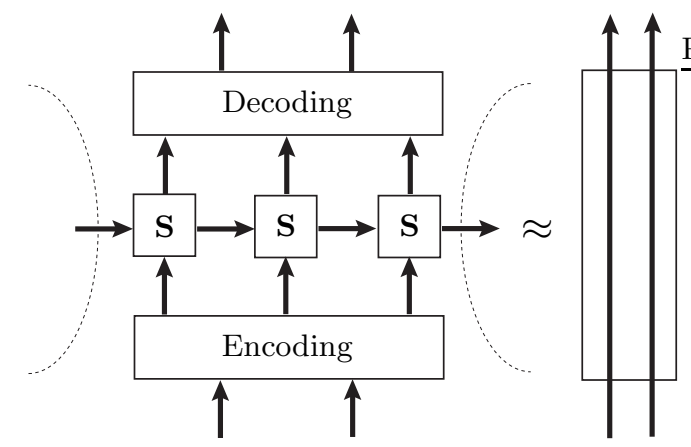

FIG. 3: Two signal states are encoded into three input registers, sent through the concatenated memory channel, and then decoded into two output states. If the overall channel is (in some sense to be specified in Section IIIB) close to the ideal channel on two inputs, the transmission rate of the above scheme is $\frac{2}{3}$. Capacity is the largest such rate, in the limit of long messages and optimal encoding and decoding. In the above setup, the initial memory input can be thought of as being controlled by either the sender or a malicious third party. Similarly, the receiver may or may not be able to read out the final memory state.

and using encoding and decoding schemes asymptotically eliminating all errors. The concept is illustrated in Fig. 3.

However, when trying to send information through a concatenated memory channel, unlike in the memoryless case we also have to specify how to handle the initial and final memory state. In particular, we may distinguish between setups in which Alice can access the initial memory input state and may use it for the encoding procedure, and setups in which a malicious third party (Eve, say) controls the initial memory input, and by her choice of the input state will try to prevent Alice and Bob from communicating over the channel. Likewise, we may consider setups in which either Bob or Eve control the final memory output. These distinctions will be made precise in Section 1IB They lead to slight variations in the notion of capacity, and in Section IIIC we will present several examples to show that the resulting capacities may or may not coincide. In particular, for channels with only one Kraus operator, all these capacities are the same, and equal the capacity of the ideal channel (cf. SectionЩI).

The various capacities can be bounded from above both in terms of the capacity of memoryless channels and in terms of entropic expressions. Some of these bounds will be presented. In particular, the standard mutual information and coherent information bounds familiar from the memoryless setting easily extend to memory channels (cf. Section VIA).

Forgetful channels are, in a sense to be specified in Section $\square$ close to memoryless channels. As such, they play a central role not only as the bridge between the axiomatic and the constructive approach to quantum
$\mathcal{A}$

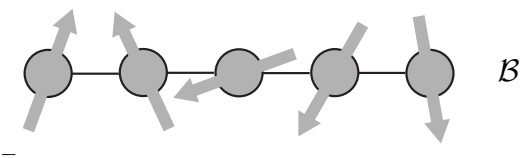

FIG. 4: An unmodulated spin chain as a quantum channel with memory: Alice places the input signal on the first spin of the chain and lets it propagate to Bob, who controls the spin at the opposite end of the chain.

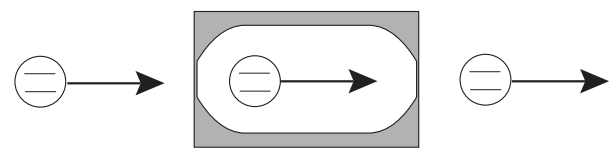

FIG. 5: In a micromaser, a stream of two-level atoms is injected into a high-quality superconducting cavity. The field modes introduce correlations between consecutive atoms.

memory channels and as generic examples for quantum memory channels, but also connect them to the memoryless realm. In Section VIB we will explain how the standard random coding techniques familiar from the memoryless setting can be modified to saturate the entropic upper bounds on the channel capacity for forgetful channels, leading to coding theorems for (private) classical and quantum information transfer for this very important class of memory channels.

We conclude with a Summary and Outlook. An Appendix contains some mathematical background relevant to the description of infinite-dimensional quantum systems, insofar as it is essential to the understanding of the Structure Theorem.

\section{B. Model Systems and Related Work}

Quantum channels which naturally acquire a memory are abundant in all branches of quantum information processing:

Recently, an unmodulated spin chain has been proposed as a model for short distance quantum communication 3 , 4, 5, 6]. In such a scheme, the state to be communicated over the channel is placed on one of the spins of the chain, propagates for a specific amount of time, and is then received at a distant spin of the chain (cf. Fig. 4). When viewed as a model for quantum communication, it is generally assumed that a reset of the spin chain occurs after each signal [7], for example by applying an external magnetic field, resulting in a memoryless channel. However, a continuous operation without reset may lead to higher transmission rates, and corresponds to a quantum channel with memory.

Another model of a quantum channel with memory is the so-called one-atom maser or micromaser [8, 9]. In such a device, excited atoms interact with the photon 
field inside a high-quality optical cavity, as depicted in Fig. 5] If the photons inside the cavity have sufficiently long lifetime, atoms entering the cavity will feel the effect of the preceding atoms, introducing correlations between consecutive signal states.

Apparently, the first model of a quantum channel with memory was introduced by Macchiavello et al. in 2001: they gave an example of a qubit channel with Markovian correlated noise [10, 11] in which entangled input states may increase the transmission rate for classical information. These results have recently been extended to some bosonic Gaussian channels [12, 13. Such an effect has been demonstrated experimentally for optical fiber channels with fluctuating birefringence, in which consecutive light pulses undergo strongly correlated polarization transformation [14, 15]. (Whether such examples exist in the memoryless setting is still an open question, and presently considered one of the most eminent open problems of quantum information theory, with wide implications for other problems in the field [16, 17].)

Subsequently, the study of quantum channels with memory has largely been confined to channels with Markovian correlated noise (cf. [18, 19] and references therein). A Lindbladian approach to memory channels has been taken by Daffer et al. 20, 21]. Upper bounds on the classical capacity for a more general class of channels have been given recently by Bowen et al. 22].

All the memory channels discussed in this Section are causal quantum channels, and thus the Structure Theorem applies. A completely different approach has been taken by Hayashi and Nagaoka [23], who refrain from imposing any structural assumption on the quantum channels they consider, and apply the information-spectrum method to obtain a coding theorem for the classical product state capacity, following work by Verdú and Han [24] on classical channels with memory.

We refer to Verdú's overview paper 25] and the GrayDavisson collection [26] for more information on memory channels in the purely classical setting.

\section{LANGUAGE AND NOTATIONS}

\section{A. States, Channels, and Observables}

According to the rules of quantum mechanics, every quantum system is associated with a Hilbert space $\mathcal{H}$, which for the purpose of this paper can mostly (but not always, see the discussion in Section IIB) be taken as finite dimensional. The observables of the system are given by bounded linear operators on the Hilbert space $\mathcal{H}$, written $\mathcal{B}(\mathcal{H})$. The physical states associated with the system are density operators $\varrho \in \mathcal{B}_{*}(\mathcal{H})$, where the latter denotes the space of trace class operators on $\mathcal{H}$.

A quantum channel $S$ which transforms input systems described by a Hilbert space $\mathcal{H}_{1}$ into output systems described by a (possibly different) Hilbert space $\mathcal{H}_{2}$ is rep- resented mathematically by a completely positive unital $\operatorname{map} S: \mathcal{B}\left(\mathcal{H}_{2}\right) \rightarrow \mathcal{B}\left(\mathcal{H}_{1}\right)$. By unitality we mean that $S\left(\mathbb{1}_{\mathcal{H}_{2}}\right)=\mathbb{1}_{\mathcal{H}_{1}}$, with the identity operator $\mathbb{1}_{\mathcal{H}_{i}} \in \mathcal{B}\left(\mathcal{H}_{i}\right)$. Each channel $S$ can be written in the so-called Kraus form [27]

$$
S(X)=\sum_{i=1}^{n} s_{i}^{*} X s_{i}
$$

with a number of $n \leq \operatorname{dim}\left(\mathcal{H}_{1}\right) \operatorname{dim}\left(\mathcal{H}_{2}\right)$ Kraus operators $s_{i}: \mathcal{H}_{1} \rightarrow \mathcal{H}_{2}$.

The physical interpretation of the quantum channel $S$ is the following: when the system is initially in the state $\varrho \in \mathcal{B}_{*}\left(\mathcal{H}_{1}\right)$, the expectation value of the measurement of the observable $X \in \mathcal{B}\left(\mathcal{H}_{2}\right)$ at the output side of the channel is given in terms of $S$ by $\operatorname{tr}(\varrho S(X))$.

Alternatively, and perhaps more intuitively, we can look at the dynamics of the states and introduce the dual map $S_{*}: \mathcal{B}_{*}\left(\mathcal{H}_{1}\right) \rightarrow \mathcal{B}_{*}\left(\mathcal{H}_{2}\right)$ by means of the duality relation

$$
\operatorname{tr}\left(S_{*}(\varrho) X\right)=\operatorname{tr}(\varrho S(X)) .
$$

$S_{*}$ is a completely positive and trace-preserving map and represents the channel in Schrödinger picture, while $S$ provides the Heisenberg picture representation (cf. Davies' textbook 28] and Keyl's survey article [2] for a more extensive discussion of observables, states, and channels).

\section{B. Heisenberg vs. Schrödinger}

For the finite dimensional systems we will consider in Section III Schrödinger picture and Heisenberg picture are completely equivalent descriptions of quantum processes by means of the duality relation Eq. (2). However, in the axiomatic characterization of quantum channels, as presented in Section IV we will have to deal with infinitedimensional systems, for which Heisenberg picture is the mandatory language. Thus, for consistency we work in Heisenberg picture throughout, emphasizing that for finite-dimensional systems conversion to Schrödinger picture is always immediate from Eq. (2). Some mathematical background on the description of infinite-dimensional systems, insofar as it is essential to the understanding of the present paper, is relegated to the Appendix. Most notably, this includes quasi-local algebras and Stinespring's dilation theorem.

\section{Distance between Quantum Channels}

From the informal discussion in Section $1 \mathrm{~A}$ it is clear that the definition of channel capacity requires the comparison of the quantum channel after the encoding and decoding process with an ideal channel. As a measure of the distance between two channels we favor the norm of 
complete boundedness, (or cb-norm, for short) [29], denoted by $\|\cdot\|_{c b}$. For two channels $T$ and $S$, the distance $\frac{1}{2}\|T-S\|_{c b}$ can be defined as the largest difference between the overall probabilities in two statistical quantum experiments differing only by exchanging one use of $S$ by one use of $T$. These experiments may involve entangling the systems on which the channels act with arbitrary further systems. Equivalently, we may set $\|T\|_{c b}=\sup _{n}\left\|T \otimes \mathrm{id}_{n}\right\|_{\infty}$, where $\|\cdot\|_{\infty}$ denotes the norm of linear operators between the Banach spaces $\mathcal{B}\left(\mathcal{H}_{i}\right)$ (cf. Appendix), and id ${ }_{n}$ denotes the identity map (ideal channel) on the $n \times n$ matrices.

Among the properties which make the cb-norm wellsuited for capacity estimates are norm multiplicativity, $\left\|T_{1} \otimes T_{2}\right\|_{c b}=\left\|T_{1}\right\|_{c b}\left\|T_{2}\right\|_{c b}$, and unitality, $\|T\|_{c b}=1$ for any channel $T$. The equivalence with other error criteria such as minimum fidelity and entanglement fidelity is discussed extensively in 1].

When working in the Schrödinger picture representation, the so-called trace norm $\|\varrho\|_{1}:=\operatorname{tr} \sqrt{\varrho^{*} \varrho}$ is frequently used to evaluate the distance between two quantum states. Again we refer to [1] for the equivalence with other distance measures.

Note that throughout this work we use base two logarithms, and we write ld $x:=\log _{2} x$.

\section{CHANNELS WITH MEMORY}

\section{A. The Constructive Approach}

A relatively simple (yet surprisingly general, see below) model to describe channels with correlated noise consists of a quantum channel which, in addition to Alice's input register system $\mathcal{H}_{A}$ and Bob's output register system $\mathcal{H}_{B}$ has an additional memory input $\mathcal{H}_{M}$ and an additional memory output $\mathcal{H}_{M^{\prime}}$. (Since the smaller of the two Hilbert space $\mathcal{H}_{M}, \mathcal{H}_{M^{\prime}}$ can always be thought of as being embedded in the larger one, in the following we will assume without loss that $\mathcal{H}_{M}=\mathcal{H}_{M^{\prime}}$.) Mathematically, a quantum channel with memory (or, for short, memory channel) is represented (in Heisenberg picture) as a completely positive and unital map $S: \mathcal{B}\left(\mathcal{H}_{B}\right) \otimes \mathcal{B}\left(\mathcal{H}_{M}\right) \rightarrow$ $\mathcal{B}\left(\mathcal{H}_{M}\right) \otimes \mathcal{B}\left(\mathcal{H}_{A}\right)$. Often we will abbreviate $\mathcal{B}\left(\mathcal{H}_{A}\right)$ to $\mathcal{A}$, and similarly for $\mathcal{B}\left(\mathcal{H}_{B}\right)$ and $\mathcal{B}\left(\mathcal{H}_{M}\right)$. Long messages with $n \in \mathbb{N}$ signal states will then be processed by subsequent application of memory channels, resulting in the concatenated channel $S_{n}: \mathcal{B}^{\otimes n} \otimes \mathcal{M} \rightarrow \mathcal{M} \otimes \mathcal{A}^{\otimes n}$ given as follows (see Fig. 1):

$$
S_{n}=\left(S \otimes \mathrm{id}_{\mathcal{A}}^{\otimes n-1}\right) \circ \ldots \circ\left(\mathrm{id}_{\mathcal{B}}^{\otimes n-2} \otimes S \otimes \mathrm{id}_{\mathcal{A}}\right) \circ\left(\mathrm{id}_{\mathcal{B}}^{n-1} \otimes S\right),
$$

where id denotes the identity operation (ideal or noiseless channel): id $(X)=X \forall X$.

The Schrödinger picture equivalent of this model was introduced by Bowen and Mancini in [19] and has been shown to encompass channels with Markovian correlated noise discussed previously in [10, 11, 14, 20]. As advertised in the Introduction, in Section IV we will show that this model is sufficiently general to describe all causal quantum channel, which was left as an open problem in [19]. However, before we prove the Structure Theorem we will extend the notion of channel capacity from the memoryless setting to channels with memory, and we will present several different setups in which these channels may be operated for the transmission of both classical and quantum information.

\section{B. Channel Capacity}

As explained in Section $\llbracket A$ the standard definition of capacity applies also to quantum channels with memory. However, as illustrated in Fig. [3] we have to specify how to handle the initial and final memory states. In particular, we need to distinguish between setups in which Alice has control over the initial memory input state and may use it for the encoding procedure, and setups in which a malicious third party (Eve, say) controls the initial memory input, and by her choice of the input state $\mu \in \mathcal{B}_{*}\left(\mathcal{H}_{M}\right)$ will try to prevent Alice and Bob from communicating over the channel. Likewise, we may consider setups in which the final memory states is either ignored or accessible to Bob, and can thus be employed in the decoding process.

In the definition of channel capacity presented below, these four different scenarios are distinguished by a different range and domain of the encoding and decoding map, respectively, and give rise to four different channel capacities for both classical and quantum information transmission.

Definition 1 Let $\mathcal{H}_{A}, \mathcal{H}_{B}$, and $\mathcal{H}_{M}$ be Hilbert spaces. $A$ positive number $R$ is called an achievable rate for the quantum memory channel $S: \mathcal{B}\left(\mathcal{H}_{B}\right) \otimes \mathcal{B}\left(\mathcal{H}_{M}\right) \rightarrow$ $\mathcal{B}\left(\mathcal{H}_{M}\right) \otimes \mathcal{B}\left(\mathcal{H}_{A}\right)$ iff for any pair of integer sequences $\left(n_{\nu}\right)_{\nu \in \mathbb{N}}$ and $\left(m_{\nu}\right)_{\nu \in \mathbb{N}}$ with $\lim _{\nu \rightarrow \infty} n_{\nu}=\infty$ and $\varlimsup_{\nu \rightarrow \infty} \frac{m_{\nu}}{n_{\nu}} \leq R$ we have

$$
\lim _{\nu \rightarrow \infty} \Delta\left(n_{\nu}, m_{\nu}\right)=0
$$

where we set

$$
\Delta\left(n_{\nu}, m_{\nu}\right):=\inf _{E, D}\left\|E S_{n_{\nu}} D-\operatorname{id}_{\mathbb{C}^{2}}^{\otimes m_{\nu}}\right\|_{c b},
$$

the infimum taken over all encoding channels $E$ and decoding channels $D$ with suitable domain and range.

The quantum channel capacity $Q(S)$ of the memory channel $S$ is defined to be the supremum of all achievable rates.

In the different setups described above, the domain of the encoding channels $E$ may or may not include the initial memory algebra $\mathcal{B}\left(\mathcal{H}_{M}\right)$, and the range of the decoding channels $D$ may or may not contain the final memory algebra $\mathcal{B}\left(\mathcal{H}_{M}\right)$, resulting in four different quantum capacities $Q_{A B}(S), Q_{A E}(S), Q_{E B, \mu}(S)$, and $Q_{E E, \mu}(S)$, where 
the first index stands for the party (ㅅlice, $\underline{B}$ ob, or $\underline{E} v e)$ who controls the initial memory state, the second index stands for the party who has access to the final memory state, and $\mu \in \mathcal{B}_{*}\left(\mathcal{H}_{M}\right)$ stands for Eve's choice of the initial memory state, if applicable.

Remark 1 The capacity of a quantum memory channel $S$ for the transmission of classical information can be defined along the same lines, restricting encoding channels to preparations and decoding channels to measurements [30], and replacing the ideal qubit channel id $\mathbb{C}^{2}$ by the ideal bit channel in Eq. (5). The respective capacities are denoted by $C_{A B}(S), C_{A E}(S), C_{E B, \mu}(S)$, and $C_{E E, \mu}(S)$, and are no smaller than their quantum counterparts.

Remark 2 In the sections to follow, we will write $Q_{*}(S)$ and $C_{*}(S)$ whenever a certain statement holds for all the four channel capacities introduced in Def. 1 regardless of Eve's choice of the initial memory state.

Remark 3 It is obvious from the definition that for every memory channel $S$ the capacities introduced in Def. [1 satisfy the following chain of inequalities:

$$
Q_{E E, \mu}(S) \leq\left\{Q_{A E}(S), Q_{E B, \mu}\right\} \leq Q_{A B}(S)
$$

for all $\mu \in \mathcal{B}_{*}\left(\mathcal{H}_{M}\right)$, and accordingly for the classical capacities $C_{E E, \mu}(S)$ etc.

Remark 4 Note that there are several equivalent definitions of channel capacity. In particular, it is sufficient to find one pair of integer sequences $\left(n_{\nu}\right)_{\nu \in \mathbb{N}}$ and $\left(m_{\nu}\right)_{\nu \in \mathbb{N}}$ such that $\varlimsup_{\nu \rightarrow \infty} \frac{m_{\nu}}{n_{\nu}}=R$ and $\lim _{\nu \rightarrow \infty} \Delta\left(n_{\nu}, m_{\nu}\right)=0$, provided the diverging sequence $\left(n_{\nu}\right)_{\nu \in \mathbb{N}}$ is subexponential, i. e., $\lim _{\nu \rightarrow \infty} \frac{n_{\nu+1}}{n_{\nu}}=1$.

In addition, the cb-norm in Eq. (5) can be replaced by other distance measures such as minimum fidelity or entanglement fidelity. See [1] for a detailed discussion of these matters.

\section{Examples}

In the following, in order to illustrate the concepts introduced above we will present several examples of quantum memory channels. These examples will also serve to show that the different capacities introduced in Def. [1 may or may not coincide, thereby justifying our defining more than one capacity.

A simple model channel for which all the capacities introduced above coincide is the Shift Channel $S^{s}$. In principle, this is just a noiseless channel, but it interchanges memory and input register: $S^{s}(b \otimes m)=b \otimes m$ (Note that in the tensor representation that we have chosen, the identity channel id comes with the inherent flip, i. e., id $(b \otimes m)=m \otimes b$.) Thus, in an $n$-fold concatenation of Shift Channels, the signals that Alice sends through the channel will be received by Bob undistorted one time-step later. In the capacity limit of long messages, as $n \rightarrow \infty$, the initial qubit that Bob may lose if Eve controls the initial memory state, and the final qubit that he may lose if he cannot access the final memory state both have a negligible impact on the transmission rate, and therefore $Q_{E E, \mu}\left(S^{s}\right)=\lim _{n \rightarrow \infty} \frac{n-2}{n} \operatorname{ld} d=\operatorname{ld} d \forall \mu \in \mathcal{B}_{*}\left(\mathcal{H}_{M}\right)$, with $d:=\operatorname{dim} \mathcal{H}_{A}=\operatorname{dim} \mathcal{H}_{B}=\operatorname{dim} \mathcal{H}_{M}$. Therefore, by Eq. (6) and Remark 10all the above capacities equal ld $d$. Further examples for channels in which the worst-case capacity and the best-case capacity are both maximal will be presented in Section IIID

An example of a memory channel in which the control over the initializing memory state can have a decisive influence on the channel performance is the channel with a global classical switch: Suppose that the memory algebra is a classical $d$-level system of diagonal $d \times d$ matrices, and that we are given a collection $\left\{T_{i}\right\}_{i=1}^{d}$ of $d$ quantum memoryless channels $T_{i}: \mathcal{B} \rightarrow \mathcal{A}$. Then a quantum memory channel $S: \mathcal{B} \otimes \mathcal{M} \rightarrow \mathcal{M} \otimes \mathcal{A}$ with a global classical switch ( $d$ settings) is given by

$$
S(b \otimes m)=\sum_{i=1}^{d}\langle i|m| i\rangle|i\rangle\langle i| \otimes T_{i}(b) .
$$

In an $n$-fold concatenation of this channel, the channel $T_{i}$ is applied in every time step if the initial memory input state was $|i\rangle\langle i|$. If Alice initially sends a pre-defined sequence of test states, Bob may find out what the initial memory setting was and choose the decoding channel accordingly. Thus, the best case capacity in this setting will be $\max _{i=1, . . d}\left\{Q\left(T_{i}\right)\right\}$, and the worst case capacity will be no larger than $\min _{i=1, . . d}\left\{Q\left(T_{i}\right)\right\}$. These two may clearly differ.

\section{Pure Channels}

Pure memory channels are channels which have only one Kraus operator in Eq. (11). From the unitality condition, $S(\mathbb{1})=\mathbb{1}$, it is then clear that these channels have a Kraus representation $S(b \otimes m)=V^{*}(b \otimes m) V$ with isometric $V: \mathcal{H}_{M} \otimes \mathcal{H}_{A} \rightarrow \mathcal{H}_{B} \otimes \mathcal{H}_{M}$.

In this section we will show that for pure channels with finite memory, the various capacities introduced in Def. 1 coincide and are maximal, i. e., we have the following

Theorem 1 Let $S: \mathcal{B}\left(\mathcal{H}_{B}\right) \otimes \mathcal{B}\left(\mathcal{H}_{M}\right) \rightarrow \mathcal{B}\left(\mathcal{H}_{M}\right) \otimes \mathcal{B}\left(\mathcal{H}_{A}\right)$ be a pure quantum memory channel with finite memory algebra $\mathcal{B}\left(\mathcal{H}_{M}\right)$. With the convention introduced in Remark $\square$ we then have:

$$
Q_{*}(S)=\min \left\{\operatorname{ld} \operatorname{dim} \mathcal{H}_{A}, \operatorname{ld} \operatorname{dim} \mathcal{H}_{B}\right\}=C_{*}(S) .
$$

Our strategy for the proof is to show that for pure channels it is possible to satisfy the Knill-Laflamme error correction criteria [31, which imply that perfect signal recovery can be achieved. This is even more than what is required for capacity purposes, since the definition of 
channel capacity, as presented in Section $\amalg$ III only demands that errors vanish asymptotically, i. e., in the limit of long messages $n \rightarrow \infty$.

Since we will have to refer to them repeatedly in the course of the proof, we start by restating the KnillLaflamme conditions for perfect error correction (cf. Th. 10.1 in [32]): A necessary and sufficient condition for a quantum channel $T: \mathcal{B}\left(\mathcal{H}_{2}\right) \rightarrow \mathcal{B}\left(\mathcal{H}_{1}\right)$ with Kraus operators $\left\{t_{i}\right\}_{i=1}^{K}$ to be completely correctable on a subspace $\mathcal{K} \subset \mathcal{H}_{1}$ is the existence of an orthonormal basis $\{|\alpha\rangle\}_{\alpha=1}^{\operatorname{dim} \mathcal{K}}$ of $\mathcal{K}$ such that

$$
\left\langle\alpha\left|t_{i}^{*} t_{j}\right| \beta\right\rangle=\omega_{i, j}\langle\alpha \mid \beta\rangle,
$$

where the coefficients $\omega_{i, j} \in \mathbb{C}$ are not permitted to depend on the basis labels $\alpha, \beta$. If the orthonormal basis $\{|\alpha\rangle\}_{\alpha} \subset \mathcal{H}_{1}$ has $N$ elements, we say that there exists a quantum code of dimension $N$.

Coming back to pure channels, we see that in the setup in which Alice controls the initial memory state and Bob can read out the final memory state there is only one (isometric) Kraus operator $V$, and thus it is straightforward to satisfy Eq. (9) and achieve rates of up to $\min \left\{\operatorname{ld} \operatorname{dim} \mathcal{H}_{A}\right.$, ld $\left.\operatorname{dim} \mathcal{H}_{B}\right\}$.

By Eq. (6) and Remark [1 in order to complete the proof of Th. 1 it is therefore sufficient to show that $Q_{E E, \mu} \geq \min \left\{\operatorname{ld} \operatorname{dim} \mathcal{H}_{A}, \operatorname{ld} \operatorname{dim} \mathcal{H}_{B}\right\} \quad \forall \mu \in \mathcal{B}_{*}\left(\mathcal{H}_{M}\right)$. Again we will show that it is possible to satisfy the errorcorrection conditions Eq. (9). However, in the worst-case scenario in which Eve chooses an arbitrary input state $\mu \in \mathcal{B}_{*}\left(\mathcal{H}_{M}\right)$ and Bob has no control over the final memory output the resulting channel is no longer pure, but can be given a Kraus representation with no more than $d_{M}^{2}$ Kraus operators, where $d_{M}:=\operatorname{dim} \mathcal{H}_{M}$ :

Lemma 2 Let $\mathcal{H}_{A}, \mathcal{H}_{B}$, and $\mathcal{H}_{M}$ be finite-dimensional Hilbert spaces, and let $d_{M}:=\operatorname{dim} \mathcal{H}_{M}$. Suppose that $S: \mathcal{B}\left(\mathcal{H}_{B}\right) \otimes \mathcal{B}\left(\mathcal{H}_{M}\right) \rightarrow \mathcal{B}\left(\mathcal{H}_{M}\right) \otimes \mathcal{B}\left(\mathcal{H}_{A}\right)$ is a pure quantum channel, i.e., $S(b \otimes m)=V^{*}(b \otimes m) V$ for isometric $V: \mathcal{H}_{M} \otimes \mathcal{H}_{A} \rightarrow \mathcal{H}_{B} \otimes \mathcal{H}_{M}$. Let $\hat{S}_{\mu}: \mathcal{B}\left(\mathcal{H}_{B}\right) \rightarrow \mathcal{B}\left(\mathcal{H}_{A}\right)$ be the restriction of $S$ to the $B$-system, with fixed initial memory state $\mu \in \mathcal{B}_{*}\left(\mathcal{H}_{M}\right)$. Then $\hat{S}_{\mu}$ can be given a Kraus representation with $d_{M}^{2}$ Kraus operators.

Proof: Let $\{|\alpha\rangle\}_{\alpha=1}^{d_{M}}$ be the eigenbasis of $\mu \in \mathcal{B}_{*}\left(\mathcal{H}_{M}\right)$, and suppose that $\{|i\rangle\}_{i=1}^{d_{A}}$ and $\left\{\left|j^{\prime}\right\rangle\right\}_{j^{\prime}=1}^{d_{B}}$ are orthonormal bases for $\mathcal{H}_{A}$ and $\mathcal{H}_{B}$, respectively. The isometry $V: \mathcal{H}_{M} \otimes \mathcal{H}_{A} \rightarrow \mathcal{H}_{B} \otimes \mathcal{H}_{M}$ can then be given the representation

$$
V=\sum_{\alpha, \beta=1}^{d_{M}} V_{\alpha, \beta} \otimes|\alpha\rangle\langle\beta|
$$

with operators $V_{\alpha, \beta}=\sum_{i=1}^{d_{A}} \sum_{j^{\prime}=1}^{d_{B}}\left\langle j^{\prime}, \alpha|V| \beta, i\right\rangle\left|j^{\prime}\right\rangle\langle i|$. From Eq. (10) we see that for arbitrary $\varrho \in \mathcal{B}_{*}\left(\mathcal{H}_{A}\right)$ and $b \in \mathcal{B}\left(\mathcal{H}_{B}\right)$ we have

$$
\begin{aligned}
\operatorname{tr}(\varrho \otimes \mu) V^{*}\left(b \otimes \mathbb{1}_{M}\right) V \\
=\sum_{\alpha, \beta, \gamma=1}^{d_{M}} \operatorname{tr}\left(\varrho V_{\alpha, \gamma}^{*} b V_{\alpha, \beta}\right)\langle\beta|\mu| \gamma\rangle \\
=\sum_{\alpha, \beta=1}^{d_{M}} \mu_{\beta} \operatorname{tr}\left(\varrho V_{\alpha, \beta}^{*} b V_{\alpha, \beta}\right) \\
=\sum_{\alpha, \beta=1}^{d_{M}} \operatorname{tr} \varrho \hat{s}_{\mu, \alpha \beta}^{*} b \hat{s}_{\mu, \alpha \beta} \\
=\operatorname{tr} \varrho \hat{S}_{\mu}(b),
\end{aligned}
$$

where we have set $\hat{s}_{\mu, \alpha \beta}:=\sqrt{\mu_{\beta}} V_{\alpha, \beta}$, and $\left\{\mu_{\beta}\right\}_{\beta=1}^{d_{M}}$ are the eigenvalues of $\mu \in \mathcal{B}_{*}\left(\mathcal{H}_{M}\right)$. Thus, the restricted channel $\hat{S}_{\mu}$ can be given a representation with $d_{M}^{2}$ Kraus operators, as claimed.

Note that in this representation the number of Kraus operators is independent of the dimension of both Alice's and Bob's systems $\mathcal{H}_{A}$ and $\mathcal{H}_{B}$, and thus the above result holds true also for the concatenated memory channel $S_{n}: \mathcal{B}\left(\mathcal{H}_{B}\right)^{\otimes n} \otimes \mathcal{B}\left(\mathcal{H}_{M}\right) \rightarrow \mathcal{B}\left(\mathcal{H}_{M}\right) \otimes \mathcal{B}\left(\mathcal{H}_{A}\right)^{\otimes n}$, independently of $n \in \mathbb{N}$. Consequently, in the limit $n \rightarrow \infty$ of long messages our setup corresponds to a channel with large input space interacting with a small environment. Physical intuition suggests that in such a setup the loss of information to the environment should be negligible, and it should be possible to operate the channel like an almost ideal one. This is the essence of the following

Lemma 3 Let $T: \mathcal{B}(\mathcal{H}) \rightarrow \mathcal{B}(\mathcal{H})$ be a channel with $K$ Kraus operators. Then there exists a quantum code of dimension at least $\left\lfloor\frac{\operatorname{dim} \mathcal{H}}{2^{K^{2}}}\right\rfloor$.

Proof: Let $\left\{t_{i}\right\}_{i=1}^{K}$ be a set of Kraus operators for $T$, and let $\tau_{i, j}:=t_{i}^{*} t_{j}$. In order to find a subspace $\mathcal{K} \subset \mathcal{H}$ of high dimensionality such that the Knill-Laflamme conditions Eq. (9) are satisfied, the following strategy may seem promising: Choose a state vector $\varphi_{1} \in \mathcal{H}$ arbitrarily, and then choose

$$
\varphi_{2} \in \mathcal{K}_{1}:=\varphi_{1}^{\perp} \cap \bigcap_{i, j=1}^{K}\left(\tau_{i, j} \varphi_{1}\right)^{\perp} .
$$

Iterate this procedure of successive removal of dimensions until no further state vectors can be found. In every step, at most $K^{2}$ dimensions are removed, so this strategy yields a subspace of dimension $\geq \frac{\operatorname{dim} \mathcal{H}}{K^{2}}$. Unfortunately, this procedure does not guarantee that inner products $\left\langle\varphi_{\alpha}\left|\tau_{i, j}\right| \varphi_{\alpha}\right\rangle$ are independent of the basis labels, as required by the Knill-Laflamme conditions Eq. (9). However, this can be accomplished by a carefully balanced pairing of eigenvectors, at the expense of a smaller code space: 
Note that any operator $\tau \in \mathcal{B}(\mathcal{H})$ can be written as the weighted sum of two Hermitian operators, $\tau=\frac{1}{2} \tau_{+}+\frac{i}{2} \tau_{-}$ with $\tau_{+}:=\tau^{*}+\tau$ and $\tau_{-}:=i\left(\tau^{*}-\tau\right)$. Since the KnillLaflamme conditions Eq. (9) are linear in the operators $\tau_{i, j}$, we may assume without loss that all operators $\tau_{i, j}$ are Hermitian. Let $\tau$ be one of these operators, and let $\left\{\lambda_{\alpha}\right\}_{\alpha=1}^{d}$ be the set of its eigenvalues, where $d:=\operatorname{dim} \mathcal{H}$ and multiple eigenvalues appear according to their multiplicity. Choose $\omega \in \mathbb{R}$ such that equally many of the real numbers $\mu_{\alpha}:=\lambda_{\alpha}-\omega$ lie on the positive and on the negative axis. (If necessary, reduce the dimension of $\mathcal{H}$ by one.) Now, if $\psi_{\alpha}$ is some eigenvector of the operator $\tau-\omega \mathbb{1}$ corresponding to the eigenvalue $\mu_{\alpha}>0$, and $\psi_{-\alpha}$ is an eigenvector corresponding to the eigenvalue $\mu_{-\alpha}<0$, by setting

$$
\varphi_{\alpha}:=\frac{1}{\sqrt{1-\frac{\mu_{\alpha}}{\mu_{-\alpha}}}}\left(\psi_{\alpha}+\sqrt{\frac{\mu_{\alpha}}{-\mu_{-\alpha}}} \psi_{-\alpha}\right),
$$

we obtain a Hilbert space $\mathcal{K}_{1}:=\operatorname{lin}\left\{\varphi_{\alpha} \mid \alpha=1, \ldots, \frac{d}{2}\right\}$ of dimension $\frac{d}{2}$ satisfying the Knill-Laflamme conditions Eq. (9) for the operator $\tau$, i. e.,

$$
\left\langle\varphi_{\alpha}|\tau-\omega \mathbb{1}| \varphi_{\beta}\right\rangle=0 \quad \forall \alpha, \beta=1, \ldots, \frac{d}{2} .
$$

Now, choose another operator $\tau^{\prime} \in\left\{\tau_{i, j}\right\}_{i, j=1}^{K}$ and repeat the above pairing procedure on the subspace $\mathcal{K}_{1}$, resulting in a subspace $\mathcal{K}_{2} \subset \mathcal{H}$ of dimension $\frac{d}{4}$. After $K^{2}$ steps, the resulting subspace has dimension at least $\frac{d}{2^{K^{2}}}$, which is the desired result.

We can now complete the proof of Theorem 1 Applying the Knill-Laflamme code described in the proof of Lemma 3 to the concatenated memory channel $\hat{S}_{\mu, n}$ with $d_{M}^{2}$ Kraus operators, we immediately see that for all $\mu \in \mathcal{B}_{*}\left(\mathcal{H}_{M}\right)$

$$
Q_{E E, \mu} \geq \lim _{n \rightarrow \infty} \frac{1}{n} \operatorname{ld} \frac{d^{n}}{2^{d_{M}^{4}}}=\operatorname{ld} d,
$$

where $d:=\min \left\{\operatorname{ld} \operatorname{dim} \mathcal{H}_{A}, \operatorname{ld} \operatorname{dim} \mathcal{H}_{B}\right\}$, as claimed.

After completion of the present work we learned that closely related results on channels interacting with small environments have been obtained independently by G. Bowen and S. Mancini [33]. These authors also show that for such channels the Knill-Laflamme error correction conditions can be fulfilled. However, instead of the pairing of eigenvalues described in the proof of Lemma 3 their approach uses convex sets arguments of Knill et al. [34], which are based on a generalization of Radon's theorem [35]. Our approach seems more straightforward, but this comes at the expense of a weaker estimate, since the more sophisticated strategy of Knill et al. yields a code of dimension $\geq \frac{d}{K^{2}\left(K^{2}+1\right)}$.

\section{THE STRUCTURE OF CAUSAL CHANNELS}

In the first part of this work we have followed a constructive approach to quantum channels with memory, in the sense that quantum channels which process long messages were always thought of as concatenations of smaller units which process one quantum signal each. In this section we take the alternative view and assume that we are a priori given a quantum channel on a long (possibly infinite) message string. Our interest is then in the internal structure of such a quantum channel. As advertised in the Introduction, we will show in Th. 团 that under very general assumptions it can be decomposed into a chain of quantum memory channels.

This result requires some mathematical background from the theory of infinite-dimensional quantum systems and channel representations, most notably quasi-local algebras and the uniqueness of the minimal Stinespring dilation. The relevant material is collected in the Appendix.

To set the stage, imagine that we have at our disposal a quantum channel which, at every discrete time step, transforms an input state on some observable algebra $\mathcal{A}$ into an output state on some (possibly different) observable algebra $\mathcal{B}$. It is represented (in Heisenberg picture) by a completely positive and unital map $T: \mathcal{B}_{\mathbb{Z}} \rightarrow \mathcal{A}_{\mathbb{Z}}$ between the quasi-local algebras $\mathcal{A}_{\mathbb{Z}}$ and $\mathcal{B}_{\mathbb{Z}}$ on Alice's and Bob's side of the channel, respectively. In the following, we will restrict ourselves to translational invariant channels, i. e., we assume that $T$ commutes with the shift on the spin chain: $\sigma_{\mathcal{A}} \circ T=T \circ \sigma_{\mathcal{B}}$. In addition, we impose the physically reasonable constraint that outputs up to some time $t$ do not depend on inputs at times $t^{\prime}>t$, leading to the following

Definition $2 A$ causal channel $T: \mathcal{B}_{\mathbb{Z}} \rightarrow \mathcal{A}_{\mathbb{Z}}$ is a completely positive and unital translational invariant map such that for every $z \in \mathbb{Z}$

$$
T\left(b_{(-\infty, z]} \otimes \mathbb{1}_{[z+1, \infty)}\right)=T\left(b_{(-\infty, z]}\right) \otimes \mathbb{1}_{[z+1, \infty)}
$$

for all $b_{(-\infty, z]} \in \mathcal{B}_{(-\infty, z]}$.

Bearing in mind that $T$ is translational invariant, we will henceforth set $z=0$, and we will use the short-hands $\mathcal{A}_{-}:=\mathcal{A}_{(-\infty, 0]}$ and $\mathcal{A}_{+}:=\mathcal{A}_{[1, \infty)}$ to denote the left and right half chain, respectively. $\mathcal{B}_{-}$and $\mathcal{B}_{+}$are defined analogously.

It is obvious from the definition that a concatenated memory channel satisfies the causality property Eq. (16). In this section we will prove the converse: every causal channel can be represented as a concatenated memory channel. Thus, we have the following Structure Theorem for causal channels (cf. Fig. 2):

Theorem 4 Let $T: \mathcal{B}_{\mathbb{Z}} \rightarrow \mathcal{A}_{\mathbb{Z}}$ be a causal channel. Ignore its outputs on the left half chain $\mathcal{B}_{-}$. Then there exists a 
memory observable algebra $\mathcal{M}$ and an initializing channel $R: \mathcal{M} \rightarrow \mathcal{A}_{-}$such that $\forall n \in \mathbb{N}$

$$
T\left(\mathbb{1}_{-} \otimes b_{n}\right)=\left(R \otimes \mathrm{id}_{\mathcal{A}}^{\otimes n}\right) S_{n}\left(b_{n} \otimes \mathbb{1}_{\mathcal{M}}\right)
$$

for all $b_{n} \in \mathcal{B}_{[1, n]} \simeq \mathcal{B}^{\otimes n}$, where $S_{n}$ is the $n$-fold concatenation of a memory channel $S: \mathcal{B} \otimes \mathcal{M} \rightarrow \mathcal{M} \otimes \mathcal{A}$, cf. Eq. (3).

Proof: In the finite-dimensional setup, a corresponding theorem has been proved by Eggeling et al. [36]. Here we generalize this result to channels on quasi-local algebras. The Appendix contains all the background information and terminology relevant to the proof of the theorem. As in the finite-dimensional setting, the uniqueness of the minimal Stinespring representation will play a crucial role.

Let $\mathcal{H}$ the Hilbert space associated with the universal representation of the left half chain $\mathcal{A}_{-}$. Note that in general $\mathcal{H}$ will not be separable. However, separability is not required in Stinespring's Theorem. Suppose that $(\mathcal{K}, \pi, V)$ is a minimal Stinespring dilation for $\left.T\right|_{\mathcal{B}_{-}}$, i. e.,

$$
T(b)=V^{*} \pi(b) V \quad \forall b \in \mathcal{B}_{-}
$$

for some Stinespring isometry $V: \mathcal{H} \rightarrow \mathcal{K}$. In the sequel, we will make repeated use of the Hilbert space isomorphism $\mathcal{H} \simeq \mathcal{H} \otimes \mathbb{C}_{d}^{\otimes n}$ (cf. Ch. 3 of Kreyszig's text [37]), where $\mathcal{A}=\mathcal{B}\left(\mathbb{C}_{d}\right)$ for some $d \in \mathbb{N}$. From Stinespring's representation Eq. (18) and the causality property Eq. (16), we may then conclude that

$$
\begin{aligned}
V^{*} \pi(b & \left.\otimes \mathbb{1}_{\mathcal{B}}^{\otimes n}\right) V=T\left(b \otimes \mathbb{1}_{\mathcal{B}}^{\otimes n}\right) \\
& =T(b) \otimes \mathbb{1}_{\mathcal{A}}^{\otimes n} \\
& =\left(V^{*} \otimes \mathbb{1}_{\mathcal{A}}^{\otimes n}\right)\left(\pi(b) \otimes \mathbb{1}_{\mathcal{A}}^{\otimes n}\right)\left(V \otimes \mathbb{1}_{\mathcal{A}}^{\otimes n}\right)
\end{aligned}
$$

for all $b \in \mathcal{B}_{-}$. Since $V$ is a minimal dilation for $T$, so is $V \otimes \mathbb{1}_{\mathcal{A}}^{\otimes n}$ for $T \otimes \mathbb{1}_{\mathcal{A}}^{\otimes n}$. As explained in Section [3 of the Appendix, we may then conclude that there exists an isometry $W_{n}: \mathcal{K} \otimes \mathbb{C}_{d}^{\otimes n} \rightarrow \mathcal{K}$ defined by

$$
W_{n}\left(\pi(b) \otimes \mathbb{1}_{\mathcal{A}}^{\otimes n}\right)\left(V \otimes \mathbb{1}_{\mathcal{A}}^{\otimes n}\right) \psi \otimes \psi_{n}:=\pi\left(b \otimes \mathbb{1}_{\mathcal{A}}^{\otimes n}\right) V \psi \otimes \psi_{n}
$$

for all $b \in \mathcal{B}_{-}, \psi \in \mathcal{H}$ and $\psi_{n} \in \mathcal{A}^{\otimes n}$ such that

$$
\pi\left(b \otimes \mathbb{1}_{\mathcal{B}}^{\otimes n}\right) W_{n}=W_{n}\left(\pi(b) \otimes \mathbb{1}_{\mathcal{A}}^{\otimes n}\right)
$$

for all $b \in \mathcal{B}_{-}$, and

$$
W_{n}\left(V \otimes \mathbb{1}_{\mathcal{A}}^{\otimes n}\right)=V .
$$

We are now in a position to reconstruct the memory algebra: Let $\mathcal{M}:=\pi^{\prime}\left(\mathcal{B}_{-}\right)$, the commutant of the observable algebra $\mathcal{B}_{-}$, and let $S_{n}: \mathcal{B}^{\otimes n} \otimes \mathcal{M} \rightarrow \mathcal{B}(\mathcal{K}) \otimes \mathcal{B}\left(\mathbb{C}_{d}^{\otimes n}\right)$ be defined by

$$
S_{n}(b \otimes m):=W_{n}^{*} \pi(b) m W_{n}
$$

for all $b \in \mathcal{B}_{-}$and $m \in \mathcal{M}$. The memory initializing channel $R: \mathcal{M} \rightarrow \mathcal{A}_{-}$is given by

$$
R(m):=V^{*} m V \quad \forall m \in \mathcal{M} .
$$

In order to justify these choices, we will first show that

$$
S_{n}\left(\mathcal{B}^{\otimes n} \otimes \mathcal{M}\right) \subset \mathcal{M} \otimes \mathcal{A}^{\otimes n} .
$$

Noting that $\pi\left(\mathbb{1}_{\mathcal{B}_{-}} \otimes \mathcal{B}^{\otimes n}\right) \mathcal{M} \subset \pi^{\prime}\left(\mathcal{B}_{-} \otimes \mathbb{1}_{B}^{\otimes n}\right)$, we see from Eq. (21) that

$$
\begin{aligned}
W_{n}^{*} & \pi\left(\mathbb{1}_{\mathcal{B}_{-}} \otimes b_{n}\right) m W_{n}\left(\pi\left(\tilde{b}_{\mathcal{B}_{-}}\right) \otimes \mathbb{1}_{A}^{\otimes n}\right) \\
& =W_{n}^{*} \pi\left(\mathbb{1}_{\mathcal{B}_{-}} \otimes b_{n}\right) m \pi\left(\tilde{b}_{\mathcal{B}_{-}} \otimes \mathbb{1}_{B}^{\otimes n}\right) W_{n} \\
& =W_{n}^{*} \pi\left(\tilde{b}_{\mathcal{B}_{-}} \otimes \mathbb{1}_{B}^{\otimes n}\right) \pi\left(\mathbb{1}_{\mathcal{B}_{-}} \otimes b_{n}\right) m W_{n} \\
& =\left(\pi\left(\tilde{b}_{\mathcal{B}_{-}}\right) \otimes \mathbb{1}_{A}^{\otimes n}\right) W_{n}^{*} \pi\left(\mathbb{1}_{\mathcal{B}_{-}} \otimes b_{n}\right) m W_{n}
\end{aligned}
$$

for all $b_{n} \in \mathcal{B}^{\otimes n}$ and $\tilde{b}_{\mathcal{B}_{-}} \in \mathcal{B}_{-}$, implying that

$$
\left[S_{n}\left(b_{n} \otimes m\right) \mid \pi\left(\tilde{b}_{\mathcal{B}_{-}}\right) \otimes \mathbb{1}_{\mathcal{A}}^{\otimes n}\right]=0,
$$

from which Eq. (25) directly follows. To complete the proof, it suffices to show that $S_{n}$ has the right concatenation properties, i. e.,

$$
\begin{aligned}
R(m) & =\left(R \otimes \operatorname{id}_{\mathcal{A}}^{\otimes n}\right) S_{n}\left(\mathbb{1}_{\mathcal{B}}^{\otimes n} \otimes m\right) \quad \text { and } \\
T(b) & =\left(R \otimes \operatorname{id}_{\mathcal{A}}^{\otimes n}\right) S_{n}\left(b \otimes \mathbb{1}_{\mathcal{M}}\right)
\end{aligned}
$$

for all $m \in \mathcal{M}$ and $b \in \mathcal{B}^{\otimes n}$. However, this is immediate from the definitions of $S_{n}$ and $R$ and Eq. (22). The result then follows by setting $S:=S_{1}$.

As can be seen from the above reasoning, the commutant algebra $\mathcal{M}$ can be replaced by the von Neumann algebra generated by all elements $\left(\right.$ id $\left.\mathcal{K} \otimes \omega_{n}\right) S_{n}\left(b_{n} \otimes \mathbb{1}_{\mathcal{M}}\right)$. However, note that in the above construction there is no unique way of choosing the memory algebra: given an infinite chain of memory channels with memory algebra $\mathcal{M}$, considering it as a causal channel and applying the memory reconstruction as in the proof of Th. 目 will in general yield a different memory algebra $\mathcal{M}^{\prime} \neq \mathcal{M}$.

It is clear from the proof of Th. 4 that the channel reconstruction will in general explicitly depend on the input initializer $R$, which describes the influence of input states in the remote past on the memory. In the following section we will turn our attention to an important class of memory channels for which the memory initializer becomes completely irrelevant. These so-called forgetful channels therefore bridge the axiomatic and the constructive approach to quantum channels with memory. We will also show that generic memory channels are forgetful.

\section{FORGETFUL CHANNELS}

Forgetful channels are quantum memory channels $S: \mathcal{B} \otimes \mathcal{M} \rightarrow \mathcal{M} \otimes A$ in which the effect of the initializing memory state dies away with time. More formally, we have the following 
Definition 3 Let $S: \mathcal{B} \otimes \mathcal{M} \rightarrow \mathcal{M} \otimes \mathcal{A}$ be a quantum memory channel, $S_{n}$ its $n$-fold concatenation, and let $\hat{S}_{n}: \mathcal{M} \rightarrow \mathcal{M} \otimes \mathcal{A}^{\otimes n}$ be the concatenated channel in which Bob's outputs are ignored: $\hat{S}_{n}(m):=S_{n}\left(\mathbb{1}_{\mathcal{B}}^{\otimes n} \otimes m\right)$ for all $m \in \mathcal{M}$. Then $S$ is called forgetful iff there exists a sequence of quantum channels $\widetilde{S}_{n}: \mathcal{M} \rightarrow \mathcal{A}^{\otimes n}$ such that

$$
\lim _{n \rightarrow \infty}\left\|\hat{S}_{n}-\mathbb{1}_{\mathcal{M}} \otimes \tilde{S}_{n}\right\|_{c b}=0 .
$$

As an illustrative example, let's consider the classically mixed channel $S:=p$ id $+(1-p) S^{s}$, where $p \in[0,1)$, and $S^{s}$ denotes the shift channel introduced in Section IIC When this channel is concatenated, in every step either the ideal channel or the shift channel is chosen with probabilities $p$ and $1-p$, respectively. The only possible way for an $n$-fold concatenation $\hat{S}_{n}$ not to be forgetful is to choose the ideal channel id in every step. However, the probability for this event is $p^{n}$, and thus vanishes in the limit $n \rightarrow \infty$, implying that Eq. (30) holds.

Remark 5 Note that Def. 3 can be relaxed by requiring only that $\left(\tilde{S}_{n}\right)_{n \in \mathbb{N}}$ is a sequence of linear maps, yet not necessarily channels. To see that this leads to an equivalent definition of forgetfulness, assume that $\left\|\hat{S}_{n}-\mathbb{1}_{\mathcal{M}} \otimes \tilde{S}_{n}\right\|_{c b} \leq \varepsilon$ for some $\varepsilon>0, n \in \mathbb{N}$, and some linear operator $\tilde{S}_{n}$. Replacing $\mathbb{1}_{\mathcal{M}} \otimes \widetilde{S}_{n}$ with the quantum channel $\left(P \otimes \mathrm{id}_{\mathcal{A}}^{\otimes n}\right) \circ \hat{S}_{n}$, where $P: \mathcal{M} \rightarrow \mathbb{C} \circ \mathbb{1}_{\mathcal{M}}$ is the completely depolarizing channel, we see that

$$
\begin{aligned}
\| \hat{S}_{n}- & \left(P \otimes \operatorname{id}_{\mathcal{A}}^{\otimes n}\right) \circ \hat{S}_{n} \|_{c b} \\
\leq & \left\|\hat{S}_{n}-\mathbb{1}_{\mathcal{M}} \otimes \tilde{S}_{n}\right\|_{c b} \\
& \quad+\left\|\left(P \otimes \operatorname{id}_{\mathcal{A}}^{\otimes n}\right) \circ\left(\mathbb{1}_{\mathcal{M}} \otimes \tilde{S}_{n}-\hat{S}_{n}\right)\right\|_{c b} \\
\leq & 2\left\|\hat{S}_{n}-\mathbb{1}_{\mathcal{M}} \otimes \tilde{S}_{n}\right\|_{c b} \leq 2 \varepsilon
\end{aligned}
$$

and thus $\lim _{n \rightarrow \infty}\left\|\hat{S}_{n}-\left(P \otimes \operatorname{id}_{\mathcal{A}}^{\otimes n}\right) \circ \hat{S}_{n}\right\|_{c b}=0$, implying that $S$ is indeed forgetful in the sense of Def. B]

There exist several equivalent criteria for a quantum memory channel to be forgetful. In particular, it is sufficient to show that the norm distance $\left\|\hat{S}_{n}-\mathbb{1}_{\mathcal{M}} \otimes \tilde{S}_{n}\right\|_{c b}$ falls below 1 for some $n \in \mathbb{N}$. What is more important, the memory effects can always be assumed to vanish exponentially fast. In addition, if the memory algebra $\mathcal{M}$ has finite dimension, the cb-norm criterion Eq. (30) can be replaced by the usual operator norm $\|\cdot\|_{\infty}$. In fact, we have the following

Proposition 5 Let $S: \mathcal{B} \otimes \mathcal{M} \rightarrow \mathcal{M} \otimes \mathcal{A}$ be a quantum memory channel, and for $n \in \mathbb{N}$ let $\hat{S}_{n}$ be defined as in Def. [3. Then $S$ is forgetful iff there exists an integer $N \in \mathbb{N}$ and some linear operator $\tilde{S}_{N}: \mathcal{M} \rightarrow \mathcal{A}^{\otimes N}$ (not necessarily a channel) such that

$$
\left\|\hat{S}_{N}-\mathbb{1}_{\mathcal{M}} \otimes \tilde{S}_{N}\right\|_{c b}<1 .
$$

Assume in addition that the memory algebra $\mathcal{M}$ has finite dimension. Then $S$ is forgetful iff for every $m \in \mathcal{M}$ and $\varepsilon>0$ we may find a positive integer $N \in \mathbb{N}$ and $a_{N} \in \mathcal{A}^{\otimes N}$ such that

$$
\left\|\hat{S}_{N}(m)-\mathbb{1}_{\mathcal{M}} \otimes a_{N}\right\|_{\infty} \leq \varepsilon\|m\|_{\infty}
$$

As advertised above, in the proof of Prop. 5 we will also be concerned with the speed of convergence in Eq. (30). In this context, the following Lemma will be helpful:

Lemma 6 Let $\left(d_{n}\right)_{n \in \mathbb{N}}$ be a positive and non-increasing sequence satisfying the subadditivity inequality

$$
d_{n+m} \leq d_{n} d_{m} \quad \forall n, m \in \mathbb{N} .
$$

Assume further that $d_{N}<1$ for some $N \in \mathbb{N}$. Then

$$
d_{n} \leq c^{n} \quad \forall n \geq N
$$

for some constant $c<1$, i. e., $\left(d_{n}\right)_{n \in \mathbb{N}}$ vanishes exponentially.

Proof of Lemma 6; Assume that $d_{N}<1$ for some $N \in \mathbb{N}$. From the subadditivity inequality (34) we then see that $d_{N+N} \leq d_{N}^{2}$, and, by induction, $d_{\nu N} \leq d_{N}^{\nu}$ for all $\nu \in \mathbb{N}$. By the monotonicity of $\left(d_{n}\right)_{n \in \mathbb{N}}$ we may then conclude that for $n \in[\nu N,(\nu+1) N)$ we have

$$
d_{n} \leq d_{\nu N} \leq d_{N}^{\nu} \leq\left(d_{N}^{\frac{1}{2 N}}\right)^{n}=c^{n}
$$

with $c:=d_{N}^{\frac{1}{2 N}}<1$, as advertised.

For the second part of the proof of Prop. [5 we obviously need to bound the cb-norm $\|\cdot\|_{c b}$ of a linear operator $R: \mathcal{B}\left(\mathcal{H}_{M}\right) \rightarrow \mathcal{A}$ with $\operatorname{dim} \mathcal{H}_{M}<\infty$ in terms of its operator norm $\|\cdot\|_{\infty}$. This is the essence of the following

Lemma 7 Let $R: \mathcal{B}\left(\mathcal{H}_{M}\right) \rightarrow \mathcal{A}$ be a linear operator, and assume that $d_{M}:=\operatorname{dim} \mathcal{H}_{M}<\infty$. We then have

$$
\|R\|_{c b} \leq d_{M}^{2}\|R\|_{\infty} .
$$

Proof of Lemma 7, By definition of the cb-norm, we have $\|R\|_{c b}=\sup _{k}\left\{\left\|R \otimes \operatorname{id}_{k}\right\|_{\infty}\right\}$, where $\operatorname{id}_{k}$ is the identity operation on the $k \times k$ matrices $\mathcal{B}\left(\mathbb{C}_{k}\right)$. Every $x \in \mathcal{B}\left(\mathcal{H}_{M}\right) \otimes \mathcal{B}\left(\mathbb{C}_{k}\right)$ can be given the expansion

$$
\begin{aligned}
x & =\sum_{\alpha} m_{\alpha} \otimes k_{\alpha} \\
& =\sum_{\alpha} \sum_{i, j=1}^{d_{M}} \mu_{\alpha, i j}|i\rangle\langle j| \otimes k_{\alpha} \\
& =\sum_{i, j=1}^{d_{M}}|i\rangle\langle j| \otimes x_{i j},
\end{aligned}
$$

where we have set $x_{i j}:=\sum_{\alpha} \mu_{\alpha, i j} k_{\alpha}$. Note that $\left\|x_{i j}\right\|_{\infty} \leq\|x\|_{\infty} \forall i, j=1, \ldots, d_{M}$, implying that

$$
\begin{aligned}
\left\|\left(R \otimes \operatorname{id}_{k}\right) x\right\|_{\infty} & =\left\|\sum_{i, j=1}^{d_{M}} R(|i\rangle\langle j|) \otimes x_{i j}\right\|_{\infty} \\
& \left.\leq \sum_{i, j=1}^{d_{M}}\|R\|_{\infty} \| i\right\rangle\left\langle j\left\|_{\infty}\right\| x_{i, j} \|_{\infty}\right. \\
& \leq d_{M}^{2}\|R\|_{\infty}\|x\|_{\infty}
\end{aligned}
$$


holds independently of $k$. Consequently, we have $\|R\|_{c b}=\sup _{k}\left\{\left\|R \otimes \mathrm{id}_{k}\right\|_{\infty}\right\} \leq d_{M}^{2}\|R\|_{\infty}$, as claimed.

We now have the necessary tools at hand to tackle the

Proof of Prop. 5: We will first prove the first part of Prop. [5] Thus, at this point we make no assumptions on the dimensionality of $\mathcal{M}$. If $S$ is forgetful, Eq. (32) is immediate from the definition. In order to prove the converse, let

$d_{n}:=\inf \left\{\left\|\hat{S}_{n}-\mathbb{1}_{\mathcal{M}} \otimes \tilde{S}_{n}\right\|_{c b} \mid \tilde{S}_{n}: \mathcal{M} \rightarrow \mathcal{A}^{\otimes n}\right.$, linear $\}$.

for $n \in \mathbb{N}$. Our strategy is to show that $\left(d_{n}\right)_{n \in \mathbb{N}}$ satisfies the conditions of Lemma 6. From Eq. (32) we can then conclude that $d_{n} \leq c^{n}$ for all $n \geq N$ for some constant $c<1$, and thus $S$ is forgetful with exponentially vanishing errors by Remark [5]

We start by showing that $\left(d_{n}\right)_{n \in \mathbb{N}}$ is non-increasing, i. e., $d_{n+1} \leq d_{n} \forall n \in \mathbb{N}$. From the definition of $\hat{S}_{n}$, we have

$$
\begin{aligned}
\hat{S}_{n+1}= & \left(\hat{S} \otimes \mathrm{id}_{\mathcal{A}}^{\otimes n}\right) \circ \hat{S}_{n} \\
= & \left(\hat{S} \otimes \mathrm{id}_{\mathcal{A}}^{\otimes n}\right) \circ\left(\hat{S}_{n}-\mathbb{1}_{\mathcal{M}} \otimes \tilde{S}_{n}\right) \\
& \quad+\left(\hat{S} \otimes \mathrm{id}_{\mathcal{A}}^{\otimes n}\right) \circ\left(\mathbb{1}_{\mathcal{M}} \otimes \tilde{S}_{n}\right) \\
= & \left(\hat{S} \otimes \mathrm{id}_{\mathcal{A}}^{\otimes n}\right)\left(\hat{S}_{n}-\mathbb{1}_{\mathcal{M}} \otimes \tilde{S}_{n}\right)+\mathbb{1}_{\mathcal{M}} \otimes \mathbb{1}_{\mathcal{A}} \otimes \tilde{S}_{n}
\end{aligned}
$$

where in the last step we have applied the unitality of $\hat{S}$. From Eq. (41) and unitality of the cb-norm we may conclude that

$$
\begin{aligned}
d_{n+1} & \leq\left\|\hat{S}_{n+1}-\mathbb{1}_{\mathcal{M}} \otimes \mathbb{1}_{\mathcal{A}} \otimes \tilde{S}_{n}\right\|_{c b} \\
& \leq\left\|\hat{S} \otimes \mathrm{id}{ }_{\mathcal{A}}^{\otimes n}\right\|_{c b}\left\|\hat{S}_{n}-\mathbb{1}_{\mathcal{M}} \otimes \tilde{S}_{n}\right\|_{c b} \leq d_{n}
\end{aligned}
$$

just as claimed. We will now show that $d_{n+m} \leq d_{n} d_{m}$ for all $n, m \in \mathbb{N}$. Similar to the above estimate, we have

$$
\begin{aligned}
\hat{S}_{n+m}= & \left(\hat{S}_{n} \otimes \mathrm{id}_{\mathcal{A}}^{\otimes m}\right) \hat{S}_{m} \\
= & \left(\hat{S}_{n} \otimes \mathrm{id}_{\mathcal{A}}^{\otimes m}\right)\left(\hat{S}_{m}-\mathbb{1}_{\mathcal{M}} \otimes \tilde{S}_{m}\right) \\
& +\left(\hat{S}_{n} \otimes \mathrm{id}_{\mathcal{A}}^{\otimes m}\right)\left(\mathbb{1}_{\mathcal{M}} \otimes \tilde{S}_{m}\right) \\
= & {\left[\left(\hat{S}_{n}-\mathbb{1}_{\mathcal{M}} \otimes \tilde{S}_{n}\right) \otimes \mathrm{id}_{\mathcal{A}}^{\otimes m}\right]\left(\hat{S}_{m}-\mathbb{1}_{\mathcal{M}} \otimes \tilde{S}_{m}\right) } \\
& +\mathbb{1}_{\mathcal{M}} \otimes \tilde{S}_{n+m},
\end{aligned}
$$

where we have introduced the short hand

$$
\tilde{S}_{n+m}:=\mathbb{1}_{\mathcal{A}}^{\otimes n} \otimes \tilde{S}_{m}+\left(\tilde{S}_{n} \otimes \mathrm{id}{ }_{\mathcal{A}}^{\otimes m}\right)\left(\hat{S}_{m}-\mathbb{1}_{\mathcal{M}} \otimes \tilde{S}_{m}\right) .
$$

Invoking again the unitality and multiplicativity of the cb-norm, we may conclude from Eq. (43) that

$$
\begin{aligned}
& \left\|\hat{S}_{n+m}-\mathbb{1}_{\mathcal{M}} \otimes \tilde{S}_{n+m}\right\|_{c b} \\
& \quad \leq\left\|\hat{S}_{n}-\mathbb{1}_{\mathcal{M}} \otimes \tilde{S}_{n}\right\|_{c b}\left\|\hat{S}_{m}-\mathbb{1}_{\mathcal{M}} \otimes \tilde{S}_{m}\right\|_{c b} \leq d_{n} d_{m},
\end{aligned}
$$

which is the desired estimate. Note that $\tilde{S}_{n+m}$ is clearly linear and unital, but not necessarily positive. This is why we did not require the maps $\tilde{S}_{n}$ to be channels in the definition of the sequence $\left(d_{n}\right)_{n \in \mathbb{N}}$. This completes the first part of the proof.

For the second part, assume that $\mathcal{M}=\mathcal{B}\left(\mathcal{H}_{M}\right)$ with $d_{M}:=\operatorname{dim} \mathcal{H}_{M}<\infty$. If Eq. (33) holds, by the same reasoning as in Remark[5]we may conclude that $\mathbb{1}_{\mathcal{M}} \otimes a_{N}$ may be replaced by $\left(P \otimes \mathrm{id}_{\mathcal{A}}^{\otimes N}\right) \circ \hat{S}_{N}(m)$, implying that for every $m \in \mathcal{M}$ and $\varepsilon>0$ we may find a positive integer $N \in \mathbb{N}$ such that

$$
\left\|\hat{S}_{N}(m)-\left(P \otimes \mathrm{id}_{\mathcal{A}}^{\otimes N}\right) \circ \hat{S}_{N}(m)\right\|_{\infty} \leq 2 \varepsilon\|m\|_{\infty} .
$$

In order to arrive at a uniform bound, let us introduce an orthonormal basis $\{|i\rangle\}_{i=1}^{d_{M}}$ for $\mathcal{H}_{M}$. Since $\mathcal{H}_{M}$ has finite dimension, Eq. (46) holds uniformally for the basis operators $\{|i\rangle\langle j|\}_{i, j=1}^{d_{M}}$ for some possibly larger $N$. Thus, by setting $m=\sum_{i, j=1}^{d_{M}} m_{i, j}|i\rangle\langle j|$ we see that

$$
\begin{aligned}
& \left\|\hat{S}_{N}(m)-\left(P \otimes \mathrm{id}_{\mathcal{A}}^{\otimes N}\right) \circ \hat{S}_{N}(m)\right\|_{\infty} \\
& \leq \sum_{i, j=1}^{d_{M}}\left|m_{i, j}\right|\left\|\hat{S}_{N}(|i\rangle\langle j|)-\left(P \otimes \operatorname{id}_{\mathcal{A}}^{\otimes N}\right) \circ \hat{S}_{N}(|i\rangle\langle j|)\right\|_{\infty} \\
& \quad \leq 2 \varepsilon \sum_{i, j=1}^{d_{M}}\left|m_{i, j}\right| \leq 2 \varepsilon d_{M}^{2}\|m\|_{\infty},
\end{aligned}
$$

where in the last step we have used that $\left|m_{i, j}\right| \leq\|m\|_{\infty}$ for all $i, j=1, \ldots, d_{M}$. Making use of Lemma 7 we may conclude from Eq. (47) that

$$
\left\|\hat{S}_{N}-\left(P \otimes \operatorname{id}_{\mathcal{A}}^{\otimes N}\right) \circ \hat{S}_{N}\right\|_{c b} \leq 2 \varepsilon d_{M}^{4} .
$$

Thus, choosing $\varepsilon<\frac{1}{2 d_{M}^{4}}$, we may find an integer $N \in \mathbb{N}$ such that Eq. (32) holds. Therefore, $S$ is forgetful by the first part of the proof. The converse is immediate from the definition of forgetfulness.

From the proof of Prop. 5 we may immediately deduce the following

Corollary 8 Let $S: \mathcal{B} \otimes \mathcal{M} \rightarrow \mathcal{M} \otimes \mathcal{A}$ be a forgetful quantum channel. Then the effect of the initial memory vanishes exponentially fast, i. e., we may find a constant $c<1$ such that

$$
\left\|\hat{S}_{n}-(P \otimes \mathrm{id} \underset{\mathcal{A}}{\otimes n}) \circ \hat{S}_{n}\right\|_{c b}<c^{n}
$$

for all sufficiently large $n$.

For convenience, and because we will use it later in SectionVI in the following Proposition we show how the definition of forgetfulness translates into the Schrödinger picture language.

Proposition 9 Let $S: \mathcal{B} \otimes \mathcal{M} \rightarrow \mathcal{M} \otimes \mathcal{A}$ be a quantum channel. Let $\varepsilon>0$, and for $n \in \mathbb{N}$ let $\hat{S}_{n}$ be defined as in Def. 3. Assume that

$$
\left\|\hat{S}_{n}-(P \otimes \mathrm{id} \underset{\mathcal{A}}{\otimes n}) \hat{S}_{n}\right\|_{\infty} \leq \varepsilon
$$


where $P: \mathcal{M} \rightarrow \mathbb{C}_{\mathcal{M}}$ is a completely depolarizing channel. We then have

$$
\left\|\operatorname{tr}_{\mathcal{B} \otimes n} S_{n *}\left(\varrho_{1}-\varrho_{2}\right)\right\|_{1} \leq 2 \varepsilon
$$

for all density operators $\varrho_{1}, \varrho_{2} \in \mathcal{M}_{*} \otimes \mathcal{A}_{*}^{\otimes n}$ such that $\operatorname{tr}_{\mathcal{M}} \varrho_{1}=\operatorname{tr}_{\mathcal{M}} \varrho_{2}$.

Conversely, suppose that Eq. (51) holds. Then Eq. (50) holds with the substitution $\varepsilon \mapsto 2 \varepsilon$.

In particular, if the quantum channel $S$ is forgetful, then from Remark 5 we know that the condition in Eq. (50) is satisfied, and thus Eq. (51) holds. If in addition the memory algebra $\mathcal{M}$ is finite-dimensional, Eq. (50) is a necessary and sufficient criterion for forgetfulness by Prop.15 By the above Proposition, Eq. (51) then gives a necessary and sufficient criterion for forgetfulness in the Schrödinger picture language.

Proof of Prop.9. Note that for any linear operator $T: \mathcal{B} \rightarrow \mathcal{A}$, the operator norm $\|T\|_{\infty}$ equals the norm of the adjoint operator on the dual space, i. e.,

$$
\|T\|_{\infty}=\sup _{\|\varrho\|_{1} \leq 1}\left\|T_{*}(\varrho)\right\|_{1}
$$

(cf. Ch. VI of [38] or Section 2.4 of [39] for details). Suppose that Eq. (50) holds. Since id ${ }_{\mathcal{A}_{*}}^{\otimes n} \otimes P_{*}=\operatorname{tr}_{\mathcal{M}}$, the partial trace on the memory algebra $\mathcal{M}$, we may conclude from Eq. (150) and the norm duality Eq. (152) that

$$
\left\|\hat{S}_{n *}(\varrho)-\hat{S}_{n *} \operatorname{tr}_{\mathcal{M}} \varrho\right\|_{1} \leq \varepsilon \forall \varrho \in \mathcal{M}_{*} \otimes \mathcal{A}_{*}^{\otimes n},
$$

which implies that for arbitrary $\varrho_{1}, \varrho_{2} \in \mathcal{M}_{*} \otimes \mathcal{A}_{*}^{\otimes n}$ such that $\operatorname{tr}_{\mathcal{M}} \varrho_{1}=\operatorname{tr}_{\mathcal{M}} \varrho_{2}$ we have

$$
\left\|\hat{S}_{n *}\left(\varrho_{1}\right)-\hat{S}_{n *}\left(\varrho_{2}\right)\right\|_{1} \leq 2 \varepsilon
$$

by application of the triangle inequality. Eq. (51) then follows by noting that $\hat{S}_{n *}=\operatorname{tr}_{\mathcal{B} \otimes n} \circ S_{n *}$.

Conversely, from Eq. (51) we can conclude that

$$
\left\|\hat{S}_{n *}\left(\varrho-\operatorname{tr}_{\mathcal{M}} \varrho\right)\right\|_{1} \leq 2 \varepsilon \quad \forall \varrho \in \mathcal{M}_{*} \otimes \mathcal{A}_{*}^{\otimes n},
$$

which implies Eq. (50) (with the substitution $\varepsilon \mapsto 2 \varepsilon$ ) by means of the norm duality Eq. (52).

Prop. 5 (and its Schrödinger dual Prop. 9) can be employed to test whether a given quantum memory channel is forgetful. As an illustrating example, let us consider the unitary partial flip operation

$$
U_{\eta}:=\cos \eta \mathbb{F}+i \sin \eta \mathbb{1}
$$

with $\eta \in[0,2 \pi)$, where $\mathbb{F}:=\sum_{i, j}|i j\rangle\langle j i|$ denotes the socalled flip operator. Since $\mathbb{F}(b \otimes m) \mathbb{F}=m \otimes b$, for $\eta=0$ the partial flip is just the Shift Channel $S^{s}$ introduced in Section $\amalg$ IIC which we know is forgetful. With the help of Prop. [5 we will show that the partial flip is forgetful whenever $\cos \eta>\frac{7}{8}$. In fact, it is sufficient to prove that

$$
\left\|U_{\eta}-\mathbb{F}\right\|_{\infty}<\frac{1}{2}
$$

holds in the designated parameter range, since this will immediately imply that

$$
\left\|U_{\eta}^{*} \mathbb{1}_{\mathcal{B}} \otimes(\cdot) U_{\eta}-\mathbb{F} \mathbb{1}_{\mathcal{B}} \otimes(\cdot) \mathbb{F}\right\|_{c b}<1,
$$

from which forgetfulness of the partial flip follows by Prop. 5] To see that Eq. (57) holds, set $\Delta_{\eta}:=U_{\eta}-\mathbb{F}$ and observe that

$$
\left\|\Delta_{\eta}^{*} \Delta_{\eta}\right\|_{\infty}=2(1-\cos \eta)<\frac{1}{4} \Longleftrightarrow \cos \eta>\frac{7}{8} .
$$

It seems likely that the partial flip is in fact forgetful over the whole parameter range, apart from $\eta=\frac{1}{2} \pi$ and $\eta=\frac{3}{2} \pi$. Evidence for this conjecture comes from the investigation of so-called collision models by Ziman et al. [40, 41], who could show forgetfulness of the partial flip when the input is restricted to product states $\varrho^{\otimes n}$.

We will prove below that forgetful quantum channels are dense in the set of quantum memory channels: for every non-forgetful quantum channel we may find a forgetful memory channel which differs arbitrarily little from it. Thus, even the partial flip at $\eta=\frac{1}{2} \pi$ and $\eta=\frac{3}{2} \pi$ (i. e., the identity $\mathbb{1}$ ) can be approximated by a forgetful quantum channel, though not necessarily a unitary one.

What is more, along the lines of the example presented above Prop. 5 can be applied to show that all quantum channels in a finite-size neighborhood of a given forgetful quantum channel are likewise forgetful, i. e., the set of forgetful quantum channels is open. Combined with the denseness of forgetful quantum channels, this justifies the claim made in Section $1 \mathrm{~A}$ that generic quantum memory channels are forgetful:

Theorem 10 The set of forgetful quantum channels is open and dense in the set of quantum memory channels in $\|\cdot\|_{c b}$-norm topology.

Proof: We will first show that the set of forgetful quantum channels is dense in the set of quantum memory channels. From any given (not necessarily forgetful) memory channel $S: \mathcal{B} \otimes \mathcal{M} \rightarrow \mathcal{M} \otimes \mathcal{A}$ we can easily construct a forgetful channel by mixing it with the completely depolarizing channel

$$
D(b \otimes m):=\operatorname{tr}((b \otimes m) \delta) \mathbb{1}_{M \otimes A},
$$

where $\delta \in \mathcal{B}_{*} \otimes \mathcal{M}_{*}$ is an arbitrary quantum state. Just as in the classically mixed shift channel discussed above, all the terms in an $n$-fold concatenation of the mixed channel $S^{\varepsilon}:=(1-\varepsilon) S+\varepsilon D$ yield the identity operator $\mathbb{1}_{\mathcal{M}}$ in the memory input, possibly apart from the $S_{n}$-contribution, which scales as $(1-\varepsilon)^{n}$, and thus vanishes as $n \rightarrow \infty$. Since this holds for all $\varepsilon>0$, and $\left\|S-S^{\varepsilon}\right\|_{c b} \leq 2 \varepsilon$, we have found a forgetful channel $S^{\varepsilon}$ arbitrarily close to $S$, completing the proof.

We will now show that the set of forgetful quantum channels is open. So assume that we are given a forgetful memory channel $S: \mathcal{B} \otimes \mathcal{M} \rightarrow \mathcal{M} \otimes \mathcal{A}$. We will show that $S$ has a finite-size neighborhood in which all memory channels are forgetful. Clearly, by the definition of 
forgetfulness we can find $N \in \mathbb{N}$ and a quantum channel $\tilde{S}_{N}: \mathcal{M} \rightarrow \mathcal{A}^{\otimes N}$ such that $\left\|\hat{S}_{N}-\mathbb{1}_{\mathcal{M}} \otimes \tilde{S}_{N}\right\|_{c b}<\frac{1}{2}$. Thus, for all memory channels $T$ such that $\|T-S\|_{c b} \leq \frac{1}{2 N}$ we have

$$
\left\|\hat{T}_{N}-\mathbb{1}_{\mathcal{M}} \otimes \tilde{S}_{N}\right\|_{c b} \leq\left\|\hat{S}_{N}-\mathbb{1}_{\mathcal{M}} \otimes \tilde{S}_{N}\right\|_{c b}+N\|T-S\|_{c b}<1,
$$

and the forgetfulness of $T$ immediately follows from Prop. 5

It is instructive to observe that a forgetful channel is obtained from a possibly non-forgetful one in the denseness proof of Th. 10 by adding a tiny amount of white noise. In real-world experiments, such noise will always be present at some level. Therefore, quantum channels encountered in the laboratory will generally be forgetful.

However, while every non-forgetful quantum channel can be approximated by a forgetful memory channel to arbitrary degree of accuracy, their capacities may be different. As an example for such a discontinuity effect, consider the channel with a global classical switch introduced in Section IIIC Let us assume that Alice and Bob face a situation in which Eve controls the initial memory state and completely jams the communication. Then adding a little bit of noise, as in the proof of Th. 10 will deprive Eve of her control of the initial memory, and may lead to a channel with positive transmission rate. Thus, adding noise may actually be beneficial sometimes. Of course, it is just as easy to construct examples of memory channels which are rendered useless by adding a tiny amount of noise.

In the special case of unitary quantum channels asymptotically vanishing memory effects have been investigated by Wellens et al. [42] under the name asymptotic completeness, with a special focus on the preparation of arbitrary memory output states. While asymptotic completeness and forgetfulness are certainly related concepts, they seem to differ in fine points, for instance in the choice of the operator topology. Asymptotic completeness of the Jaynes-Cummings interaction, which governs the physics of the micromaser experiment described in Section [B is claimed as a main mathematical result in [2]. However, a proof is neither available in the cited literature [43], nor upon request [44].

\section{ENTROPIC BOUNDS AND CHANNEL CODING}

While in Section III C and Section IIID we have computed the channel capacity of some interesting model channels, in this section we will be concerned with statements that apply more generally. In Section VIA we will give entropic upper bounds on the capacity for classical and quantum information transfer. In Section VIB achievability of these bounds will be demonstrated for forgetful quantum channels.

\section{A. Entropic Bounds}

It has already been pointed out by Bowen and Mancini 19] that the standard mutual information bound (or Holevo bound) 45] on the classical channel capacity as well as the coherent information bound [46, 47, 48, 49] on the quantum capacity can be extended to quantum channels with memory. In fact, these bounds ultimately depend only on the mutual information between Alice's input register and Bob's output register, and are independent of the internal structure of the quantum channel that links both parties. The proofs familiar from the memoryless setting can therefore be directly applied to memory channels, and yield entropic upper bounds on the classical and quantum capacity of a quantum memory channel in all the four different settings discussed in Def. 1

Before we state these bounds in Props. 11 and 12 below, we will need to introduce some notation and terminology. In the following, the von Neumann entropy of a quantum state $\varrho \in \mathcal{B}_{*}(\mathcal{H})$ will be denoted by $H(\varrho):=-\operatorname{tr}(\varrho \operatorname{ld} \varrho)$. Given a quantum channel (in Schrödinger picture) $S_{*}: \mathcal{B}_{*}\left(\mathcal{H}_{1}\right) \rightarrow \mathcal{B}_{*}\left(\mathcal{H}_{2}\right)$ and an ensemble $\left\{p_{i}, \varrho_{i}\right\}_{i=1}^{I}$ of quantum states $\varrho_{i} \in \mathcal{B}_{*}\left(\mathcal{H}_{1}\right)$, where $\left\{p_{i}\right\}_{i=1}^{I}$ is a classical probability distribution, Holevo's $\chi$-quantity is given by

$$
\chi\left(S_{*},\left\{p_{i}, \varrho_{i}\right\}\right):=H\left(\sum_{i=1}^{I} p_{i} S_{*}\left(\varrho_{i}\right)\right)-\sum_{i=1}^{I} p_{i} H\left(S_{*}\left(\varrho_{i}\right)\right) .
$$

The coherent information $I_{c}\left(S_{*}, \varrho\right)$ of the quantum channel $S_{*}$ with respect to a state $\varrho \in \mathcal{B}_{*}\left(\mathcal{H}_{1}\right)$ is likewise given in terms of the von Neumann entropy,

$$
I_{c}\left(S_{*}, \varrho\right):=H\left(S_{*}(\varrho)\right)-H\left(S_{*} \otimes \operatorname{id}(|\psi\rangle\langle\psi|)\right),
$$

where $\psi \in \mathcal{H}_{1} \otimes \mathcal{H}_{1}$ is a purification of the quantum state $\varrho \in \mathcal{B}_{*}\left(\mathcal{H}_{1}\right)[32$. With these notations, we have the following

Proposition 11 Let $S_{n *}$ be the $n$-fold concatenation of a quantum memory channel $S_{*}: \mathcal{B}_{*}\left(\mathcal{H}_{M}\right) \otimes \mathcal{B}_{*}\left(\mathcal{H}_{A}\right) \rightarrow$ $\mathcal{B}_{*}\left(\mathcal{H}_{B}\right) \otimes \mathcal{B}_{*}\left(\mathcal{H}_{M}\right)$. The classical information capacities of $S$ are bounded from above as follows:

$$
\begin{aligned}
C_{A B}(S) & \leq \varlimsup_{n \rightarrow \infty} \frac{1}{n} \max _{\left\{p_{i}, \varrho_{i}\right\}} \chi\left(S_{n *},\left\{p_{i}, \varrho_{i}\right\}\right) \\
C_{A E}(S) & \leq \varlimsup_{n \rightarrow \infty} \frac{1}{n} \max _{\left\{p_{i}, \varrho_{i}\right\}} \chi\left(\operatorname{tr}_{\mathcal{M}} \circ S_{n *},\left\{p_{i}, \varrho_{i}\right\}\right) \\
C_{E B, \mu}(S) & \leq \varlimsup_{n \rightarrow \infty} \frac{1}{n} \max _{\left\{p_{i}, \varrho_{i}\right\}} \chi\left(S_{n *},\left\{p_{i}, \mu \otimes \varrho_{i}\right\}\right) \\
C_{E E, \mu}(S) & \leq \varlimsup_{n \rightarrow \infty} \frac{1}{n} \max _{\left\{p_{i}, \varrho_{i}\right\}} \chi\left(\operatorname{tr}_{\mathcal{M}} \circ S_{n *},\left\{p_{i}, \mu \otimes \varrho_{i}\right\}\right)
\end{aligned}
$$

where $\mu \in \mathcal{B}_{*}\left(\mathcal{H}_{M}\right)$ is Eve's initial memory state. If $d_{M}:=\operatorname{dim} \mathcal{H}_{M}<\infty$, the bounds in Eq. 64), Eq. 65. 
and in Eq. (66), Eq. (67) coincide pairwise. If the channel $S$ is forgetful, the bounds in Eq. 64, Eq. [66) and in Eq. (65), Eq. (67) coincide pairwise.

Proposition 12 The quantum information capacities of the memory channel $S$ are bounded from above as follows:

$$
\begin{aligned}
Q_{A B}(S) & \leq \varlimsup_{n \rightarrow \infty} \frac{1}{n} \max _{\varrho} I_{c}\left(S_{n *}, \varrho\right), \\
Q_{A E}(S) & \leq \varlimsup_{n \rightarrow \infty} \frac{1}{n} \max _{\varrho} I_{c}\left(\operatorname{tr}_{\mathcal{M}} \circ S_{n *}, \varrho\right), \\
Q_{E B, \mu}(S) & \leq \varlimsup_{n \rightarrow \infty} \frac{1}{n} \max _{\varrho} I_{c}\left(S_{n *}, \mu \otimes \varrho\right), \\
Q_{E E, \mu}(S) & \leq \varlimsup_{n \rightarrow \infty} \frac{1}{n} \max _{\varrho} I_{c}\left(\operatorname{tr}_{\mathcal{M}} \circ S_{n *}, \mu \otimes \varrho\right),
\end{aligned}
$$

where $\mu \in \mathcal{B}_{*}\left(\mathcal{H}_{M}\right)$ is Eve's initial memory state. If $d_{M}<\infty$, the bounds in Eq. (68), Eq. (69) and in Eq. (70), Eq. (71) coincide pairwise. If the channel $S$ is forgetful, the bounds in Eq. (68), Eq. (70) and in Eq. (69), Eq. (71) coincide pairwise.

Remark 6 Note that the bounds in Props. 11] and 12 still hold when we only require that coding is possible along some (possibly very sparse) block sequence $\left(n_{\nu}\right)_{\nu \in \mathbb{N}}$. In Def. 11 we have been more ambitious, since we have required that coding works for arbitrary block size. When this stronger version of capacity is chosen, the $\varlimsup$ lim can be replaced by $\underline{\lim }$ in Eqs. (64) through (71). While the "optimistic" and the "pessimistic" channel capacity coincide for memoryless channels [1], this is not clear for channels with memory (cf. Remark 4). For forgetful channels, equivalence does hold, as will be seen in Section VIB

Proof of Props. 11] and 12; As indicated above, the proof transfers directly from the memoryless setting. We thus refer to Holevo's original work [45] for the classical bound, and to the works of Barnum et al. [46, 47, 48] and Devetak [49] for the quantum case.

Here we only show that the bounds coincide pairwise under the additional assumption of having a memory of finite size or a forgetful channel. We will begin with the finite memory case: Note that the Holevo quantity $\chi$ decreases under quantum operations, i. e.,

$$
\chi\left(R_{*} S_{*},\left\{p_{i}, \varrho_{i}\right\}\right) \leq \chi\left(S_{*},\left\{p_{i}, \varrho_{i}\right\}\right)
$$

for any pair of quantum channels $R_{*}, S_{*}$ and any ensemble of quantum states $\left\{p_{i}, \varrho_{i}\right\}_{i}[32$. We see from Eq. (72) that

$$
\begin{aligned}
\chi\left(\operatorname{tr}_{\mathcal{M}} \circ S_{n *},\right. & \left.\left\{p_{i}, \varrho_{i}\right\}\right) \leq \chi\left(S_{n *},\left\{p_{i}, \varrho_{i}\right\}\right) \\
& \leq \chi\left(\operatorname{tr}_{\mathcal{M}} \circ S_{n *},\left\{p_{i}, \varrho_{i}\right\}\right)+2 \operatorname{ld} d_{M},
\end{aligned}
$$

where in the last step the subadditivity of von Neumann entropy has been applied 32]. From Eq. (73) it immediately follows that the bounds on $C_{A B}$ and $C_{A E}$ coincide whenever $d_{M}<\infty$. The proof for the bounds on $C_{E B, \mu}$ and $C_{E E, \mu}$ is completely analogous.

For the bounds on the quantum capacities, replace Eq. (72) by the Data Processing Inequality, i. e.,

$$
I_{c}\left(R_{*} \circ S_{*}, \varrho\right) \leq I_{c}\left(S_{*}, \varrho\right)
$$

for any two quantum channels $R_{*}$ and $S_{*}$ [32], and again apply subadditivity of von Neumann entropy.

In the forgetful setting, in addition to subadditivity of von Neumann entropy we will also need to make use of its continuity properties. In fact, by Fannes' Inequality [32, 50] we have

$$
|H(\varrho)-H(\sigma)| \leq\|\varrho-\sigma\|_{1} \operatorname{ld} d+\frac{\operatorname{ld} e}{e},
$$

where $\varrho, \sigma \in \mathcal{B}_{*}(\mathcal{H})$ are quantum states, and $d:=\operatorname{dim} \mathcal{H}$.

By the results of Prop.9 forgetfulness of the channel $S$ implies that for any $\varepsilon>0$ we may find a positive integer $m \in \mathbb{N}$ such that

$$
\left\|\operatorname{tr}_{\mathcal{B} \otimes m} S_{m *}\left(\varrho_{1}-\varrho_{2}\right)\right\|_{1} \leq \varepsilon
$$

for all density operators $\varrho_{1}, \varrho_{2} \in \mathcal{B}_{*}\left(\mathcal{H}_{M}\right) \otimes \mathcal{B}_{*}\left(\mathcal{H}_{A}\right)^{\otimes n}$ satisfying $\operatorname{tr}_{\mathcal{M}} \varrho_{1}=\operatorname{tr}_{\mathcal{M}} \varrho_{2}$. Applying Fannes' Inequality Eq. (75) and subadditivity of von Neumann entropy, we can thus conclude that for arbitrary $\mu \in \mathcal{B}_{*}\left(\mathcal{H}_{M}\right)$ and $n \in \mathbb{N}$ we have

$$
\begin{aligned}
& \chi\left(S_{n *},\left\{p_{i}, \varrho_{i}\right\}\right) \leq \chi\left(\operatorname{tr}_{\mathcal{B} \otimes m} S_{n *},\left\{p_{i}, \varrho_{i}\right\}\right)+2 m \operatorname{ld} d_{B} \\
& \leq \chi\left(\operatorname{tr}_{\mathcal{B} \otimes m} S_{n *},\left\{p_{i}, \mu \otimes \operatorname{tr}_{\mathcal{M}}\left(\varrho_{i}\right)\right\}\right)+2 m \operatorname{ld} d_{B} \\
& \quad+\frac{2 \operatorname{ld} e}{e}+2\left\|\operatorname{tr}_{\mathcal{B} \otimes m} S_{n *}\left(\varrho_{i}-\mu \otimes \operatorname{tr}_{\mathcal{M}}\left(\varrho_{i}\right)\right)\right\|_{1} \operatorname{ld} d_{B}^{n} \\
& \leq \max _{\left\{q_{j}, \sigma_{j}\right\}} \chi\left(S_{n *},\left\{q_{j}, \mu \otimes \sigma_{j}\right\}\right)+2 m \operatorname{ld} d_{B} \\
& \quad+\frac{2 \operatorname{ld} e}{e}+2 n \varepsilon \operatorname{ld} d_{B} .
\end{aligned}
$$

Maximizing over the ensemble $\left\{p_{i}, \varrho_{i}\right\}$, dividing by $n$ and letting $n \rightarrow \infty$, we may conclude from Eq. (177) that

$$
\begin{aligned}
& \varlimsup_{n \rightarrow \infty} \frac{1}{n} \max _{\left\{p_{i}, \varrho_{i}\right\}} \chi\left(S_{n *},\left\{p_{i}, \varrho_{i}\right\}\right) \\
& \leq \varlimsup_{n \rightarrow \infty} \frac{1}{n} \max _{\left\{p_{i}, \varrho_{i}\right\}} \chi\left(S_{n *},\left\{p_{i}, \mu \otimes \varrho_{i}\right\}\right)+2 \varepsilon \operatorname{ld} d_{B},
\end{aligned}
$$

implying that for every $\mu \in \mathcal{B}_{*}\left(\mathcal{H}_{M}\right)$ the bound on the classical capacity $C_{E B, \mu}$ is no smaller than the bound on the capacity $C_{A B}$. The converse estimate is immediate, since Alice can obviously choose quantum ensembles of the form $\left\{p_{i}, \mu \otimes \varrho_{i}\right\}$ if she has access to the input memory. The proof for the bounds on $C_{E E, \mu}$ and $C_{A E}$ is completely analogous, as is the proof for the quantum case.

\section{B. Coding Theorems for Forgetful Channels}

In this section we will demonstrate that for forgetful channels the entropic bounds on the classical and 
quantum channel capacities presented in Prop.11 and Prop.12 are in fact achievable rates, and the limits exist.

The idea of the proof is a reduction of the problem to the memoryless setting via a relatively simple doubleblocking procedure. To illustrate the strategy, let's start with the easy case in which there is a finite integer $m \in \mathbb{N}$ such that

$$
\hat{S}_{m}=\left(P \otimes \operatorname{id}_{\mathcal{A}}^{\otimes m}\right) \circ \hat{S}_{m},
$$

where $P: \mathcal{M} \rightarrow \mathbb{C} \mathbb{1}_{\mathcal{M}}$ is again the completely depolarizing channel. We call channels with this property strictly forgetful, and the smallest integer $m$ such that Eq. (79) is satisfied will be called the memory depth of the channel $S$. For the processing of long messages, we group the channels into blocks of length $m+l$ and ignore the outputs of the first $m$ channels of each block, while the actual coding is done for the remaining $l$ channels. Eventually we will let $l \rightarrow \infty$. When we restrict the inputs to product states of block length $m+l$, due to strict forgetfulness the output state factorizes, and the whole setup corresponds to a memoryless channel on the larger input space $\mathcal{H}_{A}^{\otimes l+m}$. For the transmission of classical information, we can then apply the standard random coding techniques of Holevo 51] and Schumacher and Westmoreland 52. Invoking subadditivity of von Neumann entropy as in Section VIA the rates $R$ which can be achieved with this coding scheme are seen to be bounded as follows:

$$
\begin{aligned}
\frac{1}{l+m} \max _{\left\{p_{i}, \varrho_{i}\right\}} \chi\left(S_{l *},\left\{p_{i}, \varrho_{i}\right\}\right)-\frac{2 m}{m+l} \operatorname{ld} d_{B} \\
\quad \leq R \leq \frac{1}{l} \max _{\left\{p_{i}, \varrho_{i}\right\}} \chi\left(S_{l *},\left\{p_{i}, \varrho_{i}\right\}\right)
\end{aligned}
$$

The claim then follows by letting $l \rightarrow \infty$. For quantum channel capacities, Devetak's coding theorem [49] can be shown to yield an analogous bound, in which the Holevo quantity is replaced by coherent information.

It turns out that we can apply the same doubleblocking strategy even if the memory channel $S$ is merely assumed to be forgetful (and no longer strictly forgetful). However, in this case the output does not completely factorize, and the error we pick up by replacing the memory channel with a memoryless channel on larger blocks grows with the number of blocks. Luckily, all memory effects can be assumed to vanish exponentially fast by Corollary 8

While in this paper we have focused on the classical and quantum channel capacities proper, Devetak's proof of the quantum channel coding theorem [49] is based on a coherentification scheme for the private classical channel capacity. The setup for private information transfer (including the definition of rates and capacity) is almost the same as for classical channel capacity, but the protocols have to satisfy the additional requirement that (almost) no information is released to the environment.

More formally, assume that a quantum channel $T_{*}: \mathcal{B}_{*}\left(\mathcal{H}_{A}\right) \rightarrow \mathcal{B}_{*}\left(\mathcal{H}_{B}\right)$ is implemented by the Stinespring isometry $V: \mathcal{H}_{A} \rightarrow \mathcal{H}_{B} \otimes \mathcal{H}_{E}$, i. e.,

$$
T_{*}(\varrho)=\operatorname{tr}_{\mathrm{E}} V \varrho V^{*} \forall \varrho \in \mathcal{B}_{*}\left(\mathcal{H}_{A}\right)
$$

(cf. Section 3 of the Appendix for details). By $T_{*}^{E}$ we then denote the channel that arises from $T_{*}$ by interchanging the roles of $\mathcal{H}_{B}$ and $\mathcal{H}_{E}$, i. e.,

$$
T_{*}^{E}(\varrho):=\operatorname{tr}_{\mathrm{B}} V \varrho V^{*} \forall \varrho \in \mathcal{B}_{*}\left(\mathcal{H}_{A}\right) .
$$

This channel describes the information flow into the environment. Privacy in Devetak's coding scheme for memoryless channels then means that for sufficiently large $n \in \mathbb{N}$ we may find an operator $\Theta \in \mathcal{B}\left(\mathcal{H}_{E}\right)^{\otimes n}$ such that

$$
\left\|\frac{1}{\nu_{E}} \sum_{k=1}^{\nu_{E}} T_{*}^{E \otimes n}\left(\varrho_{j k}\right)-\Theta\right\|_{1} \leq \varepsilon \forall j=1, \ldots, \nu_{B},
$$

where $\left\{\varrho_{j k}\right\}_{j=1, k=1}^{\nu_{B}, \nu_{E}}$ is a set of codewords, and $\nu_{B}=2^{n R}$ describes the size of the code space necessary to attain the rate $R>0$. We see from Eq. (83) that privacy is achieved by randomizing over part of the codewords, leading to smaller code spaces. Devetak could show [49] that the capacity $C^{p}(T)$ of a memoryless quantum channel $T$ for private classical information transfer is given by

$$
\begin{aligned}
C^{p}(T)=\lim _{n \rightarrow \infty} \frac{1}{n} \max _{\left\{p_{i}, \varrho_{i}\right\}}\{ & \chi\left(T^{\otimes n},\left\{p_{i}, \varrho_{i}\right\}\right) \\
& \left.-\chi\left(T^{E \otimes n},\left\{p_{i}, \varrho_{i}\right\}\right)\right\},
\end{aligned}
$$

where $\chi$ is the Holevo quantity introduced in Eq. (62).

It is a coherent version of this private classical information protocol which yields the quantum channel coding theorem. Note in particular that if $\varrho=\sum_{i} p_{i}\left|\psi_{i}\right\rangle\left\langle\psi_{i}\right|$ is a decomposition of $\varrho \in \mathcal{B}_{*}\left(\mathcal{H}_{A}\right)$ into pure states, we have

$$
I_{c}(T, \varrho)=\chi\left(T,\left\{p_{i},\left|\psi_{i}\right\rangle\left\langle\psi_{i}\right|\right\}\right)-\chi\left(T^{E},\left\{p_{i},\left|\psi_{i}\right\rangle\left\langle\psi_{i}\right|\right\}\right)
$$

by the Joint Entropy Theorem (cf. Th. 11.8 of [32]).

As described above, part of our strategy in this Section will be an extension of Devetak's coherentification protocol to forgetful quantum channels. In fact, the coherentification protocol itself applies generally and does not depend on the internal structure of the quantum channel that links the sender to the receiver and the environment. Thus, our proof of the quantum coding theorem amounts to showing that the privacy condition Eq. (83) can be satisfied for forgetful quantum channels. Consequently, in the course of the proof we will also obtain a coding theorem for the private classical information of forgetful quantum channels. We thus have the following

Theorem 13 Let $\mathcal{H}_{A}, \mathcal{H}_{B}$, and $\mathcal{H}_{M}$ be finitedimensional Hilbert spaces, and let us assume that $S_{*}: \mathcal{B}_{*}\left(\mathcal{H}_{M}\right) \otimes \mathcal{B}_{*}\left(\mathcal{H}_{A}\right) \rightarrow \mathcal{B}_{*}\left(\mathcal{H}_{B}\right) \otimes \mathcal{B}_{*}\left(\mathcal{H}_{M}\right)$ is a forgetful quantum channel. By $S_{n *}$ we denote its $n$-fold concatenation. With the convention introduced in Remark 2 , we 
then have

$\begin{aligned} C_{*}(S) & =\lim _{n \rightarrow \infty} \frac{1}{n} \max _{\left\{p_{i}, \varrho_{i}\right\}} \chi\left(S_{n *},\left\{p_{i}, \varrho_{i}\right\}\right), \\ C_{*}^{p}(S) & =\lim _{n \rightarrow \infty} \frac{1}{n} \max _{\left\{p_{i}, \varrho_{i}\right\}} \chi\left(S_{n *},\left\{p_{i}, \varrho_{i}\right\}\right)-\chi\left(S_{n *}^{E},\left\{p_{i}, \varrho_{i}\right\}\right),\end{aligned}$

$Q_{*}(S)=\lim _{n \rightarrow \infty} \frac{1}{n} \max _{\varrho} I_{c}\left(S_{n *}, \varrho\right)$.

Proof: The proof of the upper bound on the private classical capacity $C_{*}^{p}(S)$, i. e.,

$C_{A B}^{p}(S) \leq \varlimsup_{n \rightarrow \infty} \frac{1}{n} \max _{\left\{p_{i}, \varrho_{i}\right\}} \chi\left(S_{n *},\left\{p_{i}, \varrho_{i}\right\}\right)-\chi\left(S_{n *}^{E},\left\{p_{i}, \varrho_{i}\right\}\right)$

is completely analogous to the one for the memoryless case [4]. For $C_{A B}(S)$ and $Q_{A B}(S)$, corresponding results have been presented in Props. 11] and 12 To complete the proof it thus remains to show that

$$
C_{E E, \mu}(S) \geq \lim _{n \rightarrow \infty} \frac{1}{n} \max _{\left\{p_{i}, \varrho_{i}\right\}} \chi\left(S_{n *},\left\{p_{i}, \varrho_{i}\right\}\right)
$$

for all $\mu \in \mathcal{B}_{*}\left(\mathcal{H}_{M}\right)$, and that the limit on the right hand side of Eq. (90) exists, and correspondingly for $C_{E E, \mu}^{p}(S)$ and $Q_{E E, \mu}(S)$.

The definition of forgetfulness combined with Corollary 8 implies that we may find a sequence $\left(\tilde{S}_{m}\right)_{m \in \mathbb{N}}$ of quantum channels such that

$$
\left\|\hat{S}_{m}-\mathbb{1}_{\mathcal{M}} \otimes \tilde{S}_{m}\right\|_{c b} \leq c^{-m}
$$

for some constant $c>1$.

As described above for the case of strictly forgetful channels, our strategy is then to group the memory channels into blocks of length $m+l$, to ignore the outputs on the first $m$ channels of each block, and to replace the resulting channel $T_{m+l}:=\left(\hat{S}_{m} \otimes \mathrm{id}_{\mathcal{A}^{\otimes l}}\right) \circ S_{l}$ by the memoryless channel

$$
\tilde{T}_{m+l}:=\left(\mathbb{1}_{\mathcal{M}} \otimes \tilde{S}_{m} \otimes \mathrm{id}_{\mathcal{A} \otimes l}\right) \circ S_{l} .
$$

For Alice, this coding procedure means that she will have to feed the first $m$ inputs of each block of length $m+l$ with some standard state $\omega \in \mathcal{B}_{*}\left(\mathcal{H}_{A}\right)^{\otimes m}$, while she will use the remaining $l$ inputs of each block for the actual coding. Bob will ignore the first $m$ output signals of each block, and will run his decoding algorithm on the remaining $l$ signals.

Let us focus on the classical information capacity first, and assume that we have a coding scheme for the memoryless channel $\tilde{T}_{m+l}$ that achieves the rate $R \in \mathbb{R}$. By definition of capacity, this means that for every $\varepsilon>0$ there is an integer $N_{\varepsilon} \in \mathbb{N}$ such that for every $n \geq N_{\varepsilon}$ we may find a code book with $\nu:=\left\lfloor 2^{\text {nlR }}\right\rfloor$ codewords $\left\{\varrho_{j}\right\}_{j=1}^{\nu} \subset \mathcal{B}_{*}\left(\mathcal{H}_{A}\right)^{\otimes l n}$ and a corresponding observable $\left\{M_{j}\right\}_{j=1}^{\nu} \subset \mathcal{B}\left(\mathcal{H}_{B}\right)^{\otimes l n}$ such that

$$
\operatorname{tr} \tilde{T}_{m+l *}^{\otimes n}\left(\varrho_{j}\right) M_{j} \geq 1-\varepsilon \forall n \geq N_{\varepsilon},
$$

uniformly in $\left\{\varrho_{j}\right\}_{j=1}^{\nu}$. By the results of Holevo 51] and Schumacher and Westmoreland [52], such coding schemes exist for all rates $R<\frac{l}{m+l} C_{1}\left(\tilde{T}_{l}\right)$, where $C_{1}\left(\tilde{T}_{l}\right)$ denotes the product state capacity of the memoryless channel $\tilde{T}_{l}$.

For the private classical information capacity, the setting is basically the same, but the codewords $\left\{\varrho_{j k}\right\}_{j=1, k=1}^{\nu_{B}, \nu_{E}}$ carry a second index to allow for randomization, and there exists an operator $\Theta \in \mathcal{B}\left(\mathcal{H}_{E}\right)^{\otimes n l}$ such that

$$
\left\|\frac{1}{\nu_{E}} \sum_{k=1}^{\nu_{E}} \tilde{T}_{l *}^{E \otimes n}\left(\varrho_{j k}\right)-\Theta\right\|_{1} \leq \varepsilon \quad \forall j=1, \ldots, \nu_{B}
$$

(cf. Eq. 83) above). Here the size of the code is given by $\nu_{B}=\left\lfloor 2^{n l R}\right\rfloor$, and all rates $R<\frac{l}{l+m} C_{1}^{p}\left(\tilde{T}_{l}\right)$ may be achieved.

The same product coding scheme will now be applied to the concatenated memory channel $T_{m+l}$. Our objectives are to show that

(a) this coding scheme satisfies the decoding condition Eq. (93),

(b) in the case of private information transfer, the privacy condition Eq. (94) holds, and

(c) the attainable rates can be made arbitrarily close to the entropic upper bounds.

This will immediately imply the coding theorem for classical and private classical information transfer. The quantum channel coding theorem will then follow from the coherentification of the private classical protocol, as explained in detail in Devetak's original work [49].

Let us start with the decoding condition $(a)$. Assume that in $n$ blocks of length $m+l$ each, the replacement $T_{m+l} \mapsto \tilde{T}_{m+l}$ is made. Since $\left\|T_{m+l}-\tilde{T}_{m+l}\right\|_{c b} \leq c^{-m}$ for each of these blocks by Eq. (91), the concatenated channels satisfy

$$
\left\|T_{n(m+l)}-\tilde{T}_{m+l}^{\otimes n}\right\|_{c b} \leq n c^{-m} .
$$

Making use of the norm duality Eq. (52), we can conclude from Eq. (95) that

$$
\left\|T_{n(m+l) *}(\varrho)-\tilde{T}_{m+l *}^{\otimes n}(\varrho)\right\|_{1} \leq n c^{-m} .
$$

Noting that for any two quantum states $\varrho, \sigma \in \mathcal{B}_{*}(\mathcal{H})$ and any observable $\left\{M_{j}\right\}_{j=1}^{\nu} \subset \mathcal{B}(\mathcal{H})$ the inequality

$$
\|\varrho-\sigma\|_{1} \geq \sum_{j=1}^{\nu}\left|\operatorname{tr} M_{j}(\varrho-\sigma)\right|
$$

holds (cf. Th. 9.1 of 32$]$ ), we may infer from Eq. (96) that for all codewords $\left\{\varrho_{j}\right\}_{j=1}^{\nu} \subset \mathcal{B}_{*}\left(\mathcal{H}_{A}\right)^{\otimes l n}$

$$
\begin{aligned}
& \operatorname{tr} T_{n(m+l) *}\left(\varrho_{j}\right) M_{j} \\
& \quad \geq \operatorname{tr} \tilde{T}_{m+l *}^{\otimes n}\left(\varrho_{j}\right) M_{j}-\left\|T_{n(m+l) *}\left(\varrho_{j}\right)-\tilde{T}_{m+l *}^{\otimes n}\left(\varrho_{j}\right)\right\|_{1} \\
& \quad \geq \operatorname{tr} \tilde{T}_{m+l *}^{\otimes n}\left(\varrho_{j}\right) M_{j}-n c^{-m} .
\end{aligned}
$$


For $\varepsilon>0$, choose $n:=l, m:=\varepsilon l$ and $l$ sufficiently large such that Eq. (93) is satisfied. We may then conclude from Eq. (98) that

$$
\operatorname{tr} T_{l^{2}(1+\varepsilon) *}\left(\varrho_{j}\right) M_{j}>1-2 \varepsilon
$$

uniformly in $j$ for sufficiently large $l$, implying that the product channel random coding scheme leads to asymptotically vanishing errors for all rates $R<\frac{1}{1+\varepsilon} C_{1}\left(\tilde{T}_{l}\right)$ and $R<\frac{1}{1+\varepsilon} C_{1}^{p}\left(\tilde{T}_{l}\right)$, respectively.

We will now show that (b) also holds, with the same substitution $\varepsilon \mapsto 2 \varepsilon$. To this end, we note that Devetak's randomization scheme can be slightly modified to include the output memory state of each block. By this trick we may guarantee that in an $l$-fold concatenation of blocks of length $m+l$ each, even the intermediate blocks, for which no coding is done and the respective outputs are ignored, are (almost) uncorrelated with Alice's signal states.

Making again use of the error estimate for concatenated channels and the norm duality Eq. (152), we may then conclude from Eq. (94) that

$$
\begin{aligned}
& \left\|\frac{1}{\nu_{E}} \sum_{k=1}^{\nu_{E}} T_{l(m+l)}^{E}\left(\varrho_{j k}\right)-\Theta\right\|_{1} \\
& \leq\left\|\frac{1}{\nu_{E}} \sum_{k=1}^{\nu_{E}}\left[T_{l(m+l)}^{E}\left(\varrho_{j k}\right)-\tilde{T}_{m+l}^{E \otimes l}\left(\varrho_{j k}\right)\right]\right\|_{1} \\
& \quad+\left\|\frac{1}{\nu_{E}} \sum_{k=1}^{\nu_{E}} \tilde{T}_{m+l}^{E \otimes l}\left(\varrho_{j k}\right)-\Theta\right\|_{1} \\
& \leq l c^{-m}+\varepsilon=l c^{-\varepsilon l}+\varepsilon \leq 2 \varepsilon
\end{aligned}
$$

for sufficiently large $l$, as advertised. Note that without the additional randomization over the output memory, the average mutual information $\frac{1}{1^{2}} H(A: E)$ between the signal states and Eve's output states will still be small. This is due to the fact that in the above coding scheme the intermediate blocks only constitute a fraction $\varepsilon$ of the total length. However, this is in general not sufficient to conclude that a norm estimate such as Eq. (100) holds.

In order to conclude the proof, it only remains to show that $C_{1}\left(\tilde{T}_{l}\right)$ can be bounded from below in terms of $\max _{\left\{p_{i}, \varrho_{i}\right\}} \chi\left(S_{l *},\left\{p_{i}, \varrho_{i}\right\}\right)$ for large $l$, and similarly for the private classical and quantum capacities.

Applying subadditivity of von Neumann entropy and Fannes' Inequality Eq. (75), we see that

$$
\begin{aligned}
\chi\left(S_{l *},\left\{p_{i}, \varrho_{i}\right\}\right) \leq \chi\left(T_{l+\varepsilon l *},\left\{p_{i}, \varrho_{i}\right\}\right)+2 \varepsilon l \operatorname{ld} d_{B} \\
\leq \chi\left(\tilde{T}_{l+\varepsilon l *},\left\{p_{i}, \varrho_{i}\right\}\right)+2 \varepsilon l \operatorname{ld} d_{B}+\frac{2 \operatorname{ld} e}{e} \\
\quad+2 l(1+\varepsilon) \varepsilon \operatorname{ld} d_{B} \\
\leq l(1+\varepsilon) C_{1}\left(\tilde{T}_{l}\right)+2 \varepsilon l \operatorname{ld} d_{B}+\frac{2 \operatorname{ld} e}{e} \\
\quad+2 l(1+\varepsilon) \varepsilon \operatorname{ld} d_{B} .
\end{aligned}
$$

Since $C_{1}\left(\tilde{T}_{l}\right)$ has been shown to be an achievable rate for large enough $l$, we may conclude from Eq. 101) that

$$
\begin{array}{r}
C_{E E, \mu}(S) \geq \frac{1}{1+\varepsilon}\left[\varlimsup_{l \rightarrow \infty} \frac{1}{l} \max _{\left\{p_{i}, \varrho_{i}\right\}} \chi\left(S_{l *},\left\{p_{i}, \varrho_{i}\right\}\right)\right. \\
\left.-4 \varepsilon \operatorname{ld} d_{B}-2 \varepsilon^{2} \operatorname{ld} d_{B}\right] .
\end{array}
$$

Since $\varepsilon>0$ is arbitrary, Eq. (102) together with the upper bound in Prop.11] entails that

$$
C_{E E, \mu}(S)=\varlimsup_{n \rightarrow \infty} \frac{1}{n} \max _{\left\{p_{i}, \varrho_{i}\right\}} \chi\left(S_{n *},\left\{p_{i}, \varrho_{i}\right\}\right) .
$$

The coding scheme described above uses blocks of length $n_{l}:=l^{2}(1+\varepsilon)$. This is a subexponential sequence in the sense of Remark 4 and we may thus apply the One-Sequence Theorem [1] to conclude that the limit in Eq. (103) exists, implying that Eq. (86) holds. The rate estimate for the private classical and quantum capacities is completely analogous.

\section{SUMMARY AND OUTLOOK}

We have presented a general model for quantum channels with memory, and shown that under mild causality constraints every quantum process can be thought of as a concatenated memory channel (plus some memory initializer).

For these memory channels, channel capacities have been introduced along the lines familiar from the memoryless context, and it has been demonstrated that different operational setups may lead to different values of the channel capacity.

While we have concentrated on the classical and quantum channel capacities proper, it is evident that the theory may be extended to memory channels assisted by additional resources, such as entanglement and classical side communication. As seen in Section VIA entropic bounds typically depend only on the amount of information shared by sender and receiver, and not on the internal structure of the quantum channel linking these two. Coding theorems for memoryless channels can easily be extended to forgetful memory channels, as demonstrated in Section VIB They typically lead to regularized expressions for the channel capacity, which still require the solution of optimization problems in Hilbert spaces of exponentially growing dimensionality. In general, computing capacities of quantum memory channels is thus at least as challenging as for memoryless channels, with less hope for improvements.

A general study of the resulting capacity landscape is still pending. In particular, we do not yet know under which general conditions some (or all) of the channel capacities introduced in Def. [1 coincide. It may seem reasonable to conjecture that, as long as the memory system is finite-dimensional, it is irrelevant for capacity purposes whether Bob or Eve control the final memory output. While this is almost immediate for the entropic 
upper bounds on the channel capacities (cf. Prop. 11 and Prop. 12), so far we have not been able to verify this conjecture for the capacities themselves.

We have demonstrated in Section $\nabla$ that generic memory channels are forgetful, and in Section VIB we have presented coding theorems for this very important class of channels. This may seem as if it were possibly to always restrict one's attention to forgetful channels. However, the capacity of a memoryless channel is sometimes discontinuous in its parameters. So while it is always possible to approximate a given non-forgetful channel by a forgetful channel to arbitrary degree of accuracy, their capacities may be very different, as the example given in Section $\nabla$ demonstrates. This calls for a more detailed analysis of non-forgetful quantum channels and their capacities.

While we have presented several equivalent criteria for a memory channel to be forgetful (cf. Section V), we do not yet have a Structure Theorem to characterize all the non-forgetful quantum channels, nor do we have a simple test to decide whether a given memory channel is forgetful.

Apart from some relatively simple model channels, little is known so far about the channel capacity of general non-forgetful memory channels. The derivation of coding theorems in this case is likely to require universal coding schemes, with encoders and decoders independent of Eve's choice of the initial memory state. For the memory channel with a global classical switch (cf. Section IIC), universal coding schemes do exist [53]. However, this is a rather special example of a memory channel, and the general case remains very much open.

\section{Acknowledgments}

We thank Charles H. Bennett, Igor Devetak, and Andreas Winter for fruitful discussions in an enjoyable atmosphere, and Garry Bowen for informing us about his work on memory channels with small environments. Alexander S. Holevo, Dirk Schlingemann, and Mario Ziman contributed perceptive comments on the manuscript and extremely valuable suggestions. Thank yous also go to Sonia Daffer for pointing us to [21], and to Aram Harrow for sharing his insight on compound channels.

Funding from Deutsche Forschungsgemeinschaft (DFG) is gratefully acknowledged.

\section{APPENDIX}

In this section we provide some mathematical background on the description of infinite-dimensional quantum systems by quasi-local algebras, and on quantum channels between such algebras. We start with a quick summary of $\mathrm{C}^{*}$-algebra terminology, and then concentrate on those aspects which are essential to the proof of the Structure Theorem in Section [IV For an in-depth treatment we refer to the texts of Bratteli and Robinson [39], Ruelle [54], and Paulsen [29].

\section{1. $\mathbf{C}^{*}$-Algebras}

The operations making up the abstract structure of $\mathrm{C}^{*}$-algebras are inspired by those known from algebras of bounded operators $\mathcal{B}(\mathcal{H})$ on a Hilbert space $\mathcal{H}$. In fact, every such operator algebra is a $\mathrm{C}^{*}$-algebra, and conversely every abstract $\mathrm{C}^{*}$-algebra is isomorphic to a norm-closed self-adjoint algebra of bounded operators on a Hilbert space. More details on this fundamental structure theorem for $\mathrm{C}^{*}$-algebras will be provided in Section 4 below.

A $\mathrm{C}^{*}$-algebra $\mathcal{A}$ is a vector space on the complex numbers $\mathbb{C}$ which is equipped with a product $a \times b \mapsto a b$ for $a, b \in \mathcal{A}$. The product is assumed to be distributive and associative, but not necessarily commutative. In addition, $\mathcal{A}$ has an adjoint operation (also called star operation or involution) $\mathcal{A} \ni a \mapsto a^{*} \in \mathcal{A}$. This is conjugate linear (or anti-linear), i. e., $(\alpha a+\beta b)^{*}=\bar{\alpha} a^{*}+\bar{\beta} b^{*}$ for all $a, b \in \mathcal{A}$ and $\alpha, \beta \in \mathbb{C}$, and has the properties $a^{* *}=a$ and $(a b)^{*}=b^{*} a^{*}$. Physicists often write $a^{+}$or $a^{\dagger}$ instead of $a^{*}$.

Besides, there is a norm $\|\cdot\|_{\infty}$ on $\mathcal{A}$ which associates a non-negative number $\|a\|_{\infty}$ to every $a \in \mathcal{A}$ such that $\|a\|_{\infty}=0$ implies $a=0$. With respect to the algebraic properties of $\mathcal{A}$, the norm satisfies $\|\alpha a\|_{\infty}=|\alpha|\|a\|_{\infty}$, the triangle inequality $\|a+b\|_{\infty} \leq\|a\|_{\infty}+\|b\|_{\infty}$ and the product inequality $\|a b\|_{\infty} \leq\|a\|_{\infty}\|b\|_{\infty}$ for all $a, b \in \mathcal{A}$ and $\alpha \in \mathbb{C}$. In addition, we have $\left\|a^{*} a\right\|_{\infty}=\|a\|_{\infty}^{2}$.

An identity $\mathbb{1}_{\mathcal{A}}$ of a $\mathrm{C}^{*}$-algebra $\mathcal{A}$ is an element of $\mathcal{A}$ such that $\mathbb{1}_{\mathcal{A}} a=a=a \mathbb{1}_{\mathcal{A}}$ for all $a \in \mathcal{A}$. A $\mathrm{C}^{*}$-algebra can have at most one identity. However, not all algebras come equipped with an identity. The absence of an identity can complicate the structural analysis, but these complications can be avoided by embedding $\mathcal{A}$ in a larger algebra $\tilde{\mathcal{A}}$ which has an identity. Here we will always assume that $\mathcal{A}$ possesses an identity. Unless the algebra is identically zero, we then have $\left\|\mathbb{1}_{\mathcal{A}}\right\|_{\infty}=1$.

A state on the $\mathrm{C}^{*}$-algebra $\mathcal{A}$ is a linear functional $\omega: \mathcal{A} \rightarrow \mathbb{C}$ which is positive in the sense that $\omega\left(a^{*} a\right) \geq 0$ for all $a \in \mathcal{A}$ and normalized such that $\omega\left(\mathbb{1}_{\mathcal{A}}\right)=1$. If $\mathcal{A}=\mathcal{B}\left(\mathcal{H}_{A}\right)$ for some finite-dimensional Hilbert space $\mathcal{H}_{A}$, to every state $\omega$ there exists a unique density operator $\varrho_{\omega} \in \mathcal{B}_{*}\left(\mathcal{H}_{A}\right)$ such that

$$
\omega(a)=\operatorname{tr}\left(\varrho_{\omega} a\right) \quad \forall a \in \mathcal{A} .
$$

For infinite-dimensional systems, there may be states which cannot be represented as density operators in the sense of Eq. A.1.

The commutant $\mathcal{A}^{\prime}$ of a $\mathrm{C}^{*}$-algebra $\mathcal{A}$ is the set of all 
operators $a \in \mathcal{A}$ that commute with $\mathcal{A}$, i. e.,

$$
\mathcal{A}^{\prime}:=\{a \in \mathcal{A} \mid a b=b a \forall b \in \mathcal{A}\} .
$$

$\mathcal{A}^{\prime}$ is a sub-algebra of $\mathcal{A}$. If $\mathcal{A}^{\prime}=\mathcal{A}$, all operators in $\mathcal{A}$ commute, and the algebra is called Abelian. These algebras describe classical systems.

\section{Quasi-Local Algebras}

Quasi-local algebras are adapted to the description of infinitely extended quantum lattice systems. The framework discussed in this Section works for any lattice structure in any spatial dimension. In fact, it does not even require translational invariance and can be formulated for possibly different quantum (or classical) systems localized on the nodes of a finite or infinite graph. However, our interest is in the input and output signals of a causal automaton, and we may thus restrict our discussion to the simple case in which the lattice consists of a one-dimensional spin chain labelled by integers $z \in \mathbb{Z}$. To each site $z \in \mathbb{Z}$ we assign an isomorphic copy $\mathcal{A}_{z}$ of the observable algebra $\mathcal{A}$, which in our case is a finitedimensional $C^{*}$-algebra $\mathcal{B}\left(\mathcal{H}_{A}\right)$ or $\mathcal{B}\left(\mathcal{H}_{B}\right)$ of Alice's input and Bob's output system, respectively. When $\Lambda \subset \mathbb{Z}$ is a finite subset, we denote by $\mathcal{A}_{\Lambda}:=\bigotimes_{z \in \Lambda} \mathcal{A}_{z}$ the algebra of observables belonging to all sites in $\Lambda$. Whenever $\Lambda_{1} \subset \Lambda_{2}$, tensoring with the identity operator $\mathbb{1}_{\mathcal{A}}$ on $\Lambda_{2} \backslash \Lambda_{1}$ will make $\mathcal{A}_{\Lambda_{1}}$ a sub-algebra of $\mathcal{A}_{\Lambda_{2}}$. In the same way the product $a_{1} a_{2}$ of operators $a_{i} \in \mathcal{A}_{\Lambda_{i}}$ becomes a well-defined element of $\mathcal{A}_{\Lambda_{1} \cup \Lambda_{2}}$. Since tensoring with the identity $\mathbb{1}_{\mathcal{A}}$ does not change the norm, this construction yields a normed algebra of local observables. Its norm-completion is called quasi-local algebra, and will be denoted by

$$
\mathcal{A}_{\mathbb{Z}}:=\overline{\bigcup_{\Lambda \subset \mathbb{Z}} \mathcal{A}_{\Lambda}}
$$

Similarly, for infinite subsystems $\Lambda \subset \mathbb{Z}$ we define $\mathcal{A}_{\Lambda}$ as the closure of the union of all $\mathcal{A}_{\Lambda^{\prime}}$ for finite $\Lambda^{\prime} \subset \Lambda$. In particular, by $\mathcal{A}_{-}:=\mathcal{A}_{(-\infty, 0]}$ and $\mathcal{A}_{+}:=\mathcal{A}_{[1, \infty)}$ we will denote the left and right half chain, respectively.

The algebra $\mathcal{A}_{\Lambda}$ is interpreted as the algebra of physical observables for a subsystem localized in the region $\Lambda \subset \mathbb{Z}$. The quasi-local algebra then corresponds to the extended algebra of observables on the infinite spin chain $\mathbb{Z}$.

On the spin chain we introduce a shift operator $\sigma$ by setting

$$
\sigma: \mathcal{A}_{\Lambda} \rightarrow \mathcal{A}_{\Lambda+1} \quad a \simeq a \otimes \mathbb{1}_{\mathcal{A}} \mapsto \sigma(a):=\mathbb{1}_{\mathcal{A}} \otimes a \simeq a,
$$

where we have used the notation $\Lambda+1:=\{z+1 \mid z \in \Lambda\}$. The canonical extension of $\sigma$ onto the quasi-local algebra $\mathcal{A}_{\mathbb{Z}}$ is a ${ }^{*}$-automorphism on $\mathcal{A}_{\mathbb{Z}}$, and the integer powers $\left\{\sigma^{z}\right\}_{z \in \mathbb{Z}}$ represent an action of the translation group $\mathbb{Z}$ by automorphisms on $\mathcal{A}_{\mathbb{Z}}$.
As explained in Section [1 a state $\omega$ on the spin chain is a positive and normalized linear functional on $\mathcal{A}_{\mathbb{Z}}$. Equivalently, a state $\omega$ is given by a family $\left\{\omega_{\Lambda}\right\}_{\Lambda \subset \mathbb{Z}}$ of density operators on $\mathcal{A}_{\Lambda}$ for finite $\Lambda \subset \mathbb{Z}$ such that $\omega(a)=$ $\operatorname{tr}\left(\omega_{\Lambda} a\right)$ for $a \in \mathcal{A}_{\Lambda}$. The local density matrices have to satisfy the consistency condition that $\operatorname{tr}_{\Lambda_{2} \backslash \Lambda_{1}} \omega_{\Lambda_{2}}=\omega_{\Lambda_{1}}$ whenever $\Lambda_{1} \subset \Lambda_{2}$. This equivalence reflects the fact that the state of the entire spin chain is assumed to be determined by the expectation values of all observables on finite subsystems $\Lambda \subset \mathbb{Z}$.

\section{Stinespring's Representation}

Quantum channels, as introduced in Section $\amalg A$ are completely positive and unital maps $S: \mathcal{B} \rightarrow \mathcal{A}$ between observable algebras $\mathcal{B}$ and $\mathcal{A}$ attributed to physical systems. In Heisenberg picture language, they describe how observables (and thus expectation values) transform when the system under consideration undergoes a free or controlled evolution.

By Stinespring's famous representation theorem 55], for every completely positive (not necessarily unital) map $S: \mathcal{B} \rightarrow \mathcal{B}\left(\mathcal{H}_{A}\right)$ we may find a Hilbert space $\mathcal{K}$ and an isometry $V: \mathcal{H}_{A} \rightarrow \mathcal{K}$ such that

$$
S(b)=V^{*} \pi(b) V \quad \forall b \in \mathcal{B},
$$

where $\pi: \mathcal{B} \rightarrow \mathcal{B}(\mathcal{K})$ is a ${ }^{*}$-representation, i. e., a linear operator that preserves the algebraic structure in that $\pi\left(b_{1} b_{2}\right)=\pi\left(b_{1}\right) \pi\left(b_{2}\right)$ and $\pi\left(b^{*}\right)=\pi(b)^{*}$.

If the output system $\mathcal{B}$ is finite-dimensional, the representation Eq. A.5 takes the simpler form

$$
S(b)=V^{*}\left(b \otimes \mathbb{1}_{\mathcal{K}}\right) V \quad \forall b \in \mathcal{B}
$$

with the Stinespring isometry $V: \mathcal{H}_{A} \rightarrow \mathcal{H}_{B} \otimes \mathcal{K}$, where $\mathcal{B}=\mathcal{B}\left(\mathcal{H}_{B}\right)$ with $\operatorname{dim} \mathcal{H}_{B}<\infty$. By means of the duality Eq. (2), in Schrödinger picture this form of Stinespring's Theorem gives rise to the ancilla representation of the quantum channel $S_{*}$,

$$
S_{*}(\varrho)=\operatorname{tr}_{\mathcal{K}} V\left(\varrho \otimes \varrho_{0}\right) V^{*} \quad \forall \varrho \in \mathcal{B}_{*}\left(\mathcal{H}_{A}\right),
$$

where $\varrho_{0} \in \mathcal{B}(\mathcal{K})$ is a so-called ancilla state. The Kraus representation Eq. (11) follows from Eq. A.6) by introducing a basis $\left\{\psi_{i}\right\}_{i}$ in $\mathcal{K}$.

A triple $(\mathcal{K}, \pi, V)$ as obtained in Stinespring's Theorem Eq. A.5 is usually called a Stinespring representation for the channel $S$. If the closed linear span of $\pi(\mathcal{B}) V \mathcal{H}_{A}$ equals $\mathcal{K}$, the representation is called minimal. Minimal Stinespring representations are unique up to unitary equivalence, in the following sense: Assume that the quantum channel $S$ has a minimal Stinespring representation Eq. A.5 as well as a further (not necessarily minimal) one

$$
S(b)=V_{1}^{*} \pi_{1}(b) V_{1} \quad \forall b \in \mathcal{B}
$$


with another Stinespring isometry $V_{1}: \mathcal{H}_{A} \rightarrow \mathcal{K}_{1}$. Since the representation Eq. A.5 is assumed to be minimal, we conclude that $\operatorname{dim} \mathcal{K} \leq \operatorname{dim} \mathcal{K}_{1}$, and the prescription

$$
W(\pi(b) V \psi):=\pi_{1}(b) V_{1} \psi
$$

for $b \in \mathcal{B}$ and $\psi \in \mathcal{H}_{A}$ yields a well-defined isometry $W: \mathcal{K} \rightarrow \mathcal{K}_{1}$. From the definition of $W$ we find that the intertwining relation $W \pi=\pi_{1} W$ holds, implying that $W \pi(b) V=\pi_{1}(b) V_{1}$ for all $b \in \mathcal{B}$, and thus $W V=V_{1}$ by setting $b=\mathbb{1}_{\mathcal{B}}$. The uniqueness statement plays a central role in the Structure Theorem for quantum memory channels (cf. Section IV).

\section{GNS-Representation of Quantum States}

A state $\omega: \mathcal{B} \rightarrow \mathbb{C}$, as defined in Section 1 above, is a unital and positive linear map. Since the range algebra $\mathbb{C}$ is Abelian, it is even completely positive (cf. [29], Th. 3.9), and thus we may apply Stinespring's Theorem to conclude that $\omega$ can be given the representation

$$
\omega(b):=\langle\Omega|\pi(b)| \Omega\rangle \quad \forall b \in \mathcal{B},
$$

where $|\Omega\rangle:=V(1)$. Eq. A.10 is usually called the GNS-representation of quantum states, after Gelfand and Naimark [56], and Segal [57].

The GNS Theorem can be applied to prove the basic structure theorem of $\mathrm{C}^{*}$-algebras:

Theorem 14 Every $\mathrm{C}^{*}$-algebra $\mathcal{A}$ is isomorphic to a norm-closed self-adjoint algebra of bounded operators on a Hilbert space.

The idea of the proof is to construct for each state $\omega$ of $\mathcal{A}$ the corresponding GNS representation $\left(\mathcal{K}_{\omega}, \pi_{\omega}, V_{\omega}\right)$, and then to form the so-called universal representation by setting

$$
\mathcal{K}:=\bigoplus_{\omega} \mathcal{K}_{\omega} \quad \text { and } \quad \pi:=\bigoplus_{\omega} \pi_{\omega}
$$

The existence of sufficiently many states is guaranteed by the Hahn-Banach extension theorem. The details are spelled out in Section 2.3 of [39].
[1] D. Kretschmann, R. F. Werner: Tema Con Variazioni: Quantum Channel Capacity, New Jour. Phys. 6 (2004) 26 (see also quant-ph/0311037)

[2] M. Keyl: Fundamentals of Quantum Information Theory, Phys. Rep. 369 (2002) Issue 5 (quant-ph/0202122)

[3] S. Bose: Quantum Communication through an Unmodulated Spin Chain, Phys. Rev. Lett. 91 (2003) 207901 (quant-ph/0212041)

[4] D. Burgarth, S. Bose: Conclusive and Arbitrarily Perfect Quantum State Transfer Using Parallel Spin Chain Channels, quant-ph/0406112 v3 (Sept. 2004)

[5] M. Christandl, N. Datta, A. Ekert, A. J. Landahl: Perfect State Transfer in Quantum Spin Networks, Phys. Rev. Lett. 92 (2004) 187902 (quant-ph/0309131)

[6] M. Christandl, N. Datta, T. C. Dorlas, A. Ekert, A. Kay, A. J. Landahl: Perfect Transfer of Arbitrary States in Quantum Spin Networks, quant-ph/0411020 (Nov. 2004)

[7] V. Giovannetti, R. Fazio: Information-Capacity Description of Spin-Chain Correlations, quant-ph/0405110 v3 (Feb. 2005)

[8] D. Meschede, H. Walther, G. Müller: One-Atom Maser, Phys. Rev. Lett. 54 (1985) 551

[9] B. T. H. Varcoe, S. Brattke, M. Weidinger, H. Walther: Preparing Pure Photon Number States of the Radiation Field, Nature 403 (2000) 743

[10] C. Macchiavello, G. M. Palma: Entanglement-Enhanced Information Transmission over a Quantum Channel with Correlated Noise, Phys. Rev. A 65 (2002) 050301 rap. comm. (quant-ph/0107052)

[11] C. Macchiavello, G. M. Palma, S. Virmani: Transition Behavior in the Channel Capacity of Two-Qubit Channels with Memory, Phys. Rev. A 69 (2004) 010303 rap. comm. (quant-ph/0307016)

[12] N. J. Cerf, J. Clavareau, C. Macchiavello, J. Roland:
Quantum Entanglement Enhances the Capacity of Bosonic Channels with Memory, quant-ph/0412089 (Dec. 2004)

[13] G. Ruggeri, G. Soliani, V. Giovannetti, Stefano Mancini: Information Transmission through Lossy Bosonic Memory Channels, quant-ph/0502093 (Feb. 2005)

[14] J. Ball, A. Dragan, K. Banaszek: Exploiting Entanglement in Communication Channels with Correlated Noise, Phys. Rev. A 69 (2004) 042324 (quant-ph/0309148)

[15] K. Banaszek, A. Dragan, W. Wasilewski, C. Radzewicz: Experimental Demonstration of Entanglement-Enhanced Classical Communication over a Quantum Channel with Correlated Noise, Phys. Rev. Lett. 92 (2004) 257901 (quant-ph/0403024)

[16] P. Shor: Equivalence of Additivity Questions in Quantum Information Theory, Commun. Math. Phys. 246 (2004) 453 (quant-ph/0305035)

[17] A. A. Pomeransky: Strong Superadditivity of the Entanglement of Formation Follows from its Additivity, Phys. Rev. A 68 (2003) 32317 (quant-ph/0305056)

[18] M. Hamada: A Lower Bound on the Quantum Capacity of Channels with Correlated Errors, JMP 43 no. 9 (2002) 4382 (quant-ph/0201056)

[19] G. Bowen, S. Mancini: Quantum Channels with a Finite Memory, Phys. Rev. A 69 (2004) 012306 (quant-ph/0305010)

[20] S. Daffer, K. Wódkiewicz, J. K. McIver: Quantum Markov Channels for Qubits, Phys. Rev. A 67 (2003) 062312 (quant-ph/0211001)

[21] S. Daffer, K. Wódkiewicz, J. D. Cresser, J. K. McIver: Depolarizing Channel as a Completely Positive Map with Memory, Phys. Rev. A 70 (2004) 010304(R) (quant-ph/0309081)

[22] G. Bowen, S. Mancini, I. Devetak: Bounds on Classical Information Capacities for a Class of Quantum Memory 
Channels, quant-ph/0312216 v2 (April 2004)

[23] M. Hayashi, H. Nagaoka: General Formulas for Capacity of Classical-Quantum Channels, IEEE Trans. Inf. Th. 49 (2003) 1753 (quant-ph/0206186)

[24] S. Verdú, T. S. Han: A General Formula for Channel Capacity, IEEE Trans. Inf. Th. 40 (1994) 1147

[25] S. Verdú: Fifty Years of Shannon Theory, IEEE Trans. Inf. Th. 44 (1998) 2057

[26] R. Gray, L. D. Davisson: Ergodic and Information Theory (Dowden, Hutchinson \& Ross, Stroudsburg, Pennsylvania, 1977)

[27] K. Kraus: States, Effects, and Operations (Springer, Berlin, 1983)

[28] E. B. Davies: Quantum Theory of Open Systems (Academic, London, 1976)

[29] V. I. Paulsen: Completely Bounded Maps and Operator Algebras (Cambridge University Press, Cambridge, 2002)

[30] R. F. Werner: Quantum Information Theory - An Invitation, in ed. G. Alber et al., Quantum Information (Springer, Berlin, 2001), quant-ph/0101061

[31] E. Knill, R. Laflamme: Theory of Quantum ErrorCorrecting Codes, Phys. Rev. A 55 (1997) 900 (quant-ph/9604034)

[32] M. A. Nielsen, I. L. Chuang: Quantum Computation and Quantum Information (Cambridge University Press, Cambridge, 2000)

[33] G. Bowen: private communication (Sept. 2004)

[34] E. Knill, R. Laflamme, L. Viola: Theory of Quantum Error Correction for General Noise, Phys. Rev. Lett. 84 (2000) 2525 (quant-ph/9908066)

[35] H. Tverberg: A Generalization of Radon's Theorem, J. London Math. Soc. 41 (1966) 123

[36] T. Eggeling, D. Schlingemann, R. F. Werner: Semicausal Operations are Semilocalizable, Europhys. Lett. 57 (2002) 782 (quant-ph/0104027)

[37] E. Kreyszig: Introductory Functional Analysis with Applications (John Wiley, New York, 1978)

[38] M. Reed, B. Simon: Methods of Modern Mathematical Physics I: Functional Analysis (Academic, New York, 1980)

[39] O. Bratteli, D. W. Robinson: Operator Algebras and Quantum Statistical Mechanics 1, 2nd edition (Springer, Berlin, 1987)

[40] M. Ziman, P. Štelmachovic, V. Bužek, M. Hillery, V. Scarani, N. Gisin: Diluting Quantum Information: An Analysis of Information Transfer in SystemReservoir Interactions, Phys. Rev. A 65 (2002) 042105 (quant-ph/0110164)

[41] V. Scarani, M. Ziman, P. Štelmachovic, N. Gisin, V.
Bužek: Thermalizing Quantum Machines: Dissipation and Entanglement, Phys. Rev. Lett. 88 (2002) 097905 (quant-ph/0110088)

[42] T. Wellens, A. Buchleitner, B. Kümmerer, H. Maassen: Quantum State Preparation via Asymptotic Completeness, Phys. Rev. Lett. 85 (2000) 3361

[43] B. Kümmerer, H. Maassen: A Scattering Theory for Markov Chains, Infin. Dimens. Anal. Quantum Probab. Relat. Top. 3 (2000) 161

[44] B. Kümmerer: private communication (Feb. 2005)

[45] A. S. Holevo: Some Estimates for the Information Content Transmitted by a Quantum Communication Channel, Probl. Inform. Transm. 9 (1973) 3

[46] H. Barnum, M. A. Nielsen, B. Schumacher: Information Transmission through a Noisy Quantum Channel, Phys. Rev. A 57 (1998) 4153 (quant-ph/9702049)

[47] H. Barnum, J. A. Smolin, B. Terhal: Quantum Capacity is Properly Defined without Encodings, Phys. Rev. A 58 (1998) 3496 (quant-ph/9711032)

[48] H. Barnum, E. Knill, M. A. Nielsen: On Quantum Fidelities and Channel Capacities, IEEE Trans. Inf. Th. 46 (2000) 1317 (quant-ph/9809010)

[49] I. Devetak: The Private Classical Information Capacity and Quantum Information Capacity of a Quantum Channel, IEEE Trans. Inf. Th. 51 (2005) 44 (quant-ph/0304127)

[50] M. Fannes: A Continuity Property of the Entropy Density for Spin Lattice Systems, Comm. Math. Phys. 31 (1973) 291

[51] A. S. Holevo: The Capacity of the Quantum Channel with Generalized Signal States, IEEE Trans. Inf. Th. 44 (1998) 269 (quant-ph/9611023)

[52] B. Schumacher, M. Westmoreland: Sending Classical Information via Noisy Quantum Channels, Phys. Rev. A 56 (1997) 131

[53] D. Kretschmann, R. F. Werner: forthcoming publication (2005)

[54] D. Ruelle: Statistical Mechanics: Rigorous Results (Imperial College Press and World Scientific Publishing, London, 1999)

[55] W. F. Stinespring: Positive Functions on $\mathrm{C}^{*}$-Algebras, Proc. Amer. Math. Soc. 6 (1955) 211

[56] I. M. Gelfand, M. A. Naimark: On the Imbedding of Normed Rings into the Ring of Operators in Hilbert space, Mat. Sb. 12 (1943) 197

[57] I. E. Segal: Irreducible Representations of Operator Algebras, Bull. Amer. Math. Soc. 61 (1947) 69 